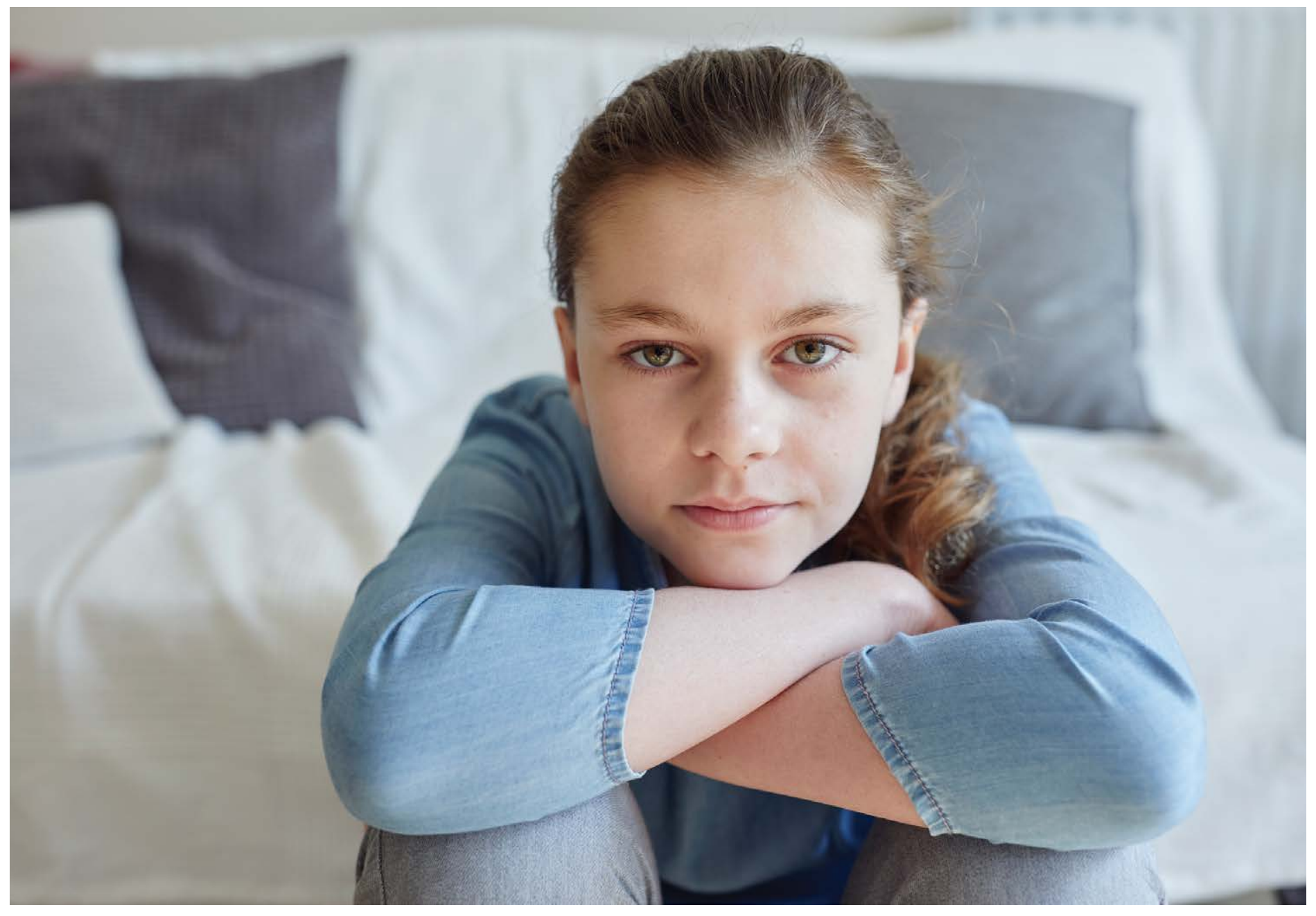

\title{
The economic costs of child maltreatment in the UK
}

A preliminary study

Gabriella Conti

Stephen Morris

Mariya Melnychuk

Elena Pizzo

University College London 


\section{Acknowledgements}

This report would not have been possible without the input of the advisory group. A huge thank you to Helen Fisher, John Devaney, Chris Cuthbert, Haroon Chowdry, Andrew James, Jon Brown, Alan Wardle, Pam Miller and Sonja Jütte for their time and input.

This research was funded by the NSPCC. 
1. Literature review on the economic impact of child maltreatment

1.1 UK Studies of Child Maltreatment

1.2 Economics Studies of Child Maltreatment

1.3 Cost Studies

2. Datasets and definition of child maltreatment 10

2.1 National Child Development Study 10

2.2 English Longitudinal Study of Ageing 12

2.3 Edinburgh Study of Youth Transitions and Crime 12

2.4 British Household Panel Study/UK Household Longitudinal Study 13

3. The effects of child maltreatment 15

3.1 National Child Development Study 16

$\begin{array}{ll}3.2 \text { English Longitudinal Study of Ageing } & 17\end{array}$

4. The long-term economic consequences of child maltreatment in the UK

4.1 Aims 28

4.2 Methods 28

Costs included in the analysis $\quad 28$

Costing methodology $\quad 29$

Lifetime costs per victim of non-fatal child maltreatment 30

Short-term health-related costs 30

Unplanned hospital admissions for injuries $\quad 30$

Short-term mental health problems $\quad 31$

Long term health-related costs $\quad 32$

Anxiety $\quad 32$

Depression $\quad 32$

Smoking 33

Alcohol abuse $\quad 33$

Criminal justice system costs 33

Child social care costs $\quad 34$

Special education costs $\quad 35$

Productivity losses due to reduced employment 36

Total costs and sensitivity analyses $\quad 36$

Lifetime costs per death from child maltreatment 37

4.3 Results 42

Lifetime costs per victim of non-fatal child maltreatment $\quad 42$

Lifetime costs per death from child maltreatment $\quad 45$ 
5. Discussion 46

Summary of findings $\quad 46$

Strengths and limitations $\quad 46$

Limitations resulting from the datasets $\quad 46$

Limitations of the cost analysis $\quad 46$

$\begin{array}{ll}\text { Comparison with other studies } & 47\end{array}$

$\begin{array}{ll}\text { Future research } & 47\end{array}$

$\begin{array}{ll}\text { Implications of this research } & 48\end{array}$

$\begin{array}{ll}\text { References } & 49\end{array}$

Appendix

UK Studies of Child Maltreatment $\quad 51$

Economics Studies of Child Maltreatment

Cost Studies 


\section{An introduction from NSPCC}

This research examines the evidence for the economic costs of child maltreatment. This study is the first UK-based study that estimates the lifetime costs of child maltreatment. It uses existing UK longitudinal datasets. The estimates reflect the financial costs of child maltreatment in terms of its impact on health care, social care, education, the criminal justice system and also the costs to the wider economy in terms of lost productivity. Very importantly, this study does not capture significant intangible costs such as the emotional suffering borne by victims.

The analysis shows that the discounted average lifetime incidence cost of non-fatal child maltreatment by a primary care-giver to be $£ 89,390$ (with a 95\% certainty that the costs fall between $£ 44,896$ and $£ 145,508^{1}$ ). The largest contributors to these costs were social care costs, short-term health-related costs, and the costs resulting from a lower probability of employment.

The NSPCC and the researchers from University College London who undertook this research consider that this study presents a conservative estimate of the economic costs of child maltreatment. The estimate was limited by the range of child maltreatment and outcome measures in the data. For example, studies identified in the literature review for this report found important costs that could not be quantified as there were no relevant variables in the longitudinal datasets (e.g. days off work, premature mortality and drug use). Further, the literature review suggested a number of outcomes were related to child maltreatment (such as obesity, hypertension, type 2 diabetes, raised cholesterol and cancer) but no effect on these was identified in the longitudinal datasets and they were therefore not included in the cost analysis.

These factors mean that this study gives us a conservative estimate of the economic costs of child maltreatment in the UK. The estimates are useful for the economic evaluation of child maltreatment interventions and prevention services and can also be useful when there is a need to prepare a business case to make the case for a service or intervention where there may not have been one to date. This study represents a baseline on which future, more comprehensive, studies might be built when better data are available. 


\section{Overview}

This document constitutes the final report for the project "Estimating the economic costs of child maltreatment in UK". According to the NSPCC (2011) child abuse includes "all forms of physical and/or emotional ill-treatment, sexual abuse, neglect or negligent treatment or commercial or other exploitation, resulting in actual or potential harm to the child's health, survival, development or dignity in the context of a relationship of responsibility, trust or power". The purpose of this research is to examine the evidence for the economic costs of child maltreatment and to quantify them as far as is possible using UK datasets. The ambition was to develop the first comprehensive estimates of the costs of child maltreatment in the UK across different short- and long-term outcomes. We used an incidence-based approach to calculate the lifetime costs of child maltreatment. Our cost estimates have focused on the financial costs of child maltreatment in terms of its impact on health care, social care, education, the criminal justice system, and the wider economy in terms of lost productivity. We have not been able to include intangible costs such as the emotional pain borne by victims, though we recognise these are substantial.

This research is important as incidence-based costs that represent the total lifetime costs resulting from cases of child maltreatment are useful for the economic evaluation of child maltreatment intervention/prevention activities. Our hope is that the findings of this report will help make the economic case for interventions to prevent and reduce child maltreatment. Further, articulating and quantifying the longer-term economic impacts can help develop and shape a wider policy narrative about child maltreatment. There are clearly costs that fall onto the individual who is maltreated; however there are also costs that fall on to society more broadly.

Our new estimates of the effects of child maltreatment relate to maltreatment by primary caregivers and encompass both different forms of maltreatment (neglect, and physical, sexual and emotional abuse) and an overall measure. They are based on representative UK data, and show that having experienced any maltreatment in childhood is associated with worse mental health outcomes, smoking behaviour, alcohol use, lower probability of employment and greater welfare dependence; no robust effects are however detected for physical health, heavy smoking and wages.

We calculated the lifetime costs per victim of non-fatal and fatal child maltreatment by primary caregivers from a societal perspective in the UK. We used the best available evidence to develop our estimates, including the findings from our statistical analysis of the effects of child maltreatment. We included costs to the health care, social care, education and criminal justice sectors, and to the wider economy in terms of lost productivity. The discounted average lifetime cost of non-fatal child maltreatment per victim was calculated to be $£ 89,390$ (95\% uncertainty interval $£ 44,896$ to $£ 145,508)$. The largest contributors to these costs were social care costs, short-term health-related costs, and reduced employment costs. Our central estimate of the discounted lifetime cost per death from child maltreatment was $£ 940,758$, comprising health care costs and lost productivity costs. There was uncertainty in our estimates so we undertook extensive sensitivity analyses. Overall, our estimates should be considered as being cautious and conservative.

The report is structured as follows. Section 1 includes a detailed review of the UK, economics, and cost literatures on the effects of child maltreatment on a variety of outcomes. Section 2 details the data sources considered for the project and the definitions of child maltreatment adopted. Section 3 presents the results of new analyses based on UK data which estimate the effect of child maltreatment on a variety of socioeconomic and health outcomes at different stages of the lifecourse. Section 4 details the cost analysis. 


\section{Literature review on the economic impact of child maltreatment}

The literature on child maltreatment is vast and interdisciplinary, encompassing different contributions of both a theoretical and applied nature from a variety of different fields, ranging from child development and psychology, to economics and legal studies. Hence, we decided to focus the review of the literature on three areas which are directly related to the aim of this project: studies focused on the UK, studies from the literature in economics, and studies undertaking cost analyses. The details on how these reviews were performed, and the criteria according to which different papers were selected, are reported in the next subsections.

\subsection{UK Studies of Child Maltreatment}

We started from an extensive review of all the literature in different disciplines based on UK data. 69 studies were initially identified through database searching, including PubMed and Google Scholar. The search terms used were: "child*hood maltreatment', 'child*hood adversity', 'child*hood abuse', 'child*hood neglect', 'adverse childhood' AND 'UK', 'England', 'British'. After initial screening, 20 studies were selected for the review, based on sample size, representativeness of the data, and relevance with the current project. Below we report the main summary of findings. The list of all the 20 papers and a table giving details on all of them is in the appendix (Table A1).

We identified three nationally representative data sources that have been previously used in the analysis of the impact of child maltreatment: the National Child Development Study (NCDS) or 1958 birth cohort, the Avon Longitudinal Study of Parents and Children (ALSPAC), and the Environmental Risk (E-Risk) Longitudinal Twin Study.

Different definitions of child maltreatment have been used in the studies based on UK data. Prospective (neglect at age 7,11 and 16) and retrospective (neglect and abuse at age 45 reporting abuse/neglect to age 16) measures were available in NCDS data, while the definition of child maltreatment varied considerably between studies. In addition, a frequently analysed measure was a cumulative score of adverse child experiences (ACE), including abuse, neglect and different combinations of other types of ACE (e.g. household dysfunction, maternal absence, parents' mental illness, parents' alcohol abuse etc.). Hence caution is needed when comparing between studies.

The following outcomes were considered across the various studies: (1) health outcomes: mental health, including psychotic symptoms, affective and anxiety disorders, adiposity, height trajectories, BMI, diabetes, pubertal development, risk of cancer, changes in DNA methylation, physiological wear-and-tear; (2) cognitive outcomes: cognitive development, education qualifications; (3) social integration: social relations and social networking. The methods used were linear regressions and logistic models. The main findings were that childhood maltreatment, especially physical and sexual abuse, was associated with higher risk of a number of health problems, both physical and mental, lower educational attainment, cognitive decline and lower enjoyment of close relationships with other people.

\subsection{Economics Studies of Child Maltreatment}

We reviewed all the economics literature on the factors affecting or the impact of child maltreatment and identified only nine papers. We included UK and non-UK papers in this review, to inform the methodology we used in our subsequent analysis. The search terms were as for the first review, except we did not limit the search to UK, English or British studies, and we focused on the economic literature only using 'econ*' as a search term and including studies published mainly in economics journals. The smaller number of papers examining the impact of child maltreatment in the economics literature as compared to other fields is likely due to the lack of suitable data and especially the difficulty of finding credible identification strategies to estimate the causal impact of maltreatment. The complete list of papers reviewed is available in the Appendix.

Among these nine papers, four estimate the impact of socioeconomic conditions on the number of cases of CM, using state-level rates of child abuse and neglect reports and number of children in foster care from the United States. These papers find that increases in the fraction of children with absent fathers and working mothers, and in the share of families with non-working parents and those with 
income below the poverty line in a state are related to increases in many measures of CM. Additionally, they find that legalized abortion led to a decline in total reported incidents of child abuse, while Medicaid funding restrictions were associated with an increase in substantiated reports.

Then, the majority of the papers estimating the effects of CM on various outcomes use data from the National Longitudinal Study of Adolescent Health (AddHealth), ${ }^{2}$ a siblings or twins fixed effects design and exactly the same variables to identify CM, in stark contrast with the UK studies, as outlined above. In this case, the CM indicators were derived from wave III, when the respondents were asked (at ages 18-26) whether and how often (before they were in 6th grade): (1) parents (or other adult caregivers) had not taken care of their basic needs, such as keeping them clean or providing food or clothing; (2) parents (or other adult caregivers) slapped, hit, or kicked them; (3) parents (or other adult caregivers) had touched them in a sexual way, forced them to touch him or her in a sexual way, or forced them to have sexual relations; (4) parents (or other adult caregivers) left them home alone when an adult should have been with them. The authors constructed indicators for "ever" of each type of harm, and also for $>10$ times for physical abuse and being left alone. These papers find long-lasting effects of CM on a variety of different outcomes, such as health, depression, personality traits, the probability of committing crime, and socioeconomic outcomes, such as education, employment, earnings and assets. The costs are greater for those who experienced multiple types of CM in particular physical and sexual abuse.

\subsection{Cost Studies}

Lastly, we carried out an extensive and comprehensive review of the cost literature in the UK and worldwide. Limited data are available on the costs associated with the initial immediate maltreatment and associated long-term costs of maltreatment in the UK.
We searched PubMed, Embase, Google Scholar using "child abuse" OR "abused children" OR "child neglect" OR "neglected children" OR "sexual abuse" OR "sexually abused children" OR "emotional abuse" OR "emotionally abused children" OR "emotional neglect" OR "physical abuse" OR "physically abused children" AND "costs and cost analysis" OR "economics" OR "public expenditure" OR "resource allocation". We searched for studies published in peer reviewed journals, reports and other publications in English, without limiting the analysis by country. We found 26 studies. The abstract of each paper or report was screened and studies were selected according to methodology, type of analysis, presence of cost analysis, relevance of the study. We then read the full text of each selected paper and extracted the relevant information needed for our study. Below we report a summary of findings. A list of all the 26 studies is in the appendix.

The most common objective of the included studies $s$ to estimate the cost of child maltreatment (in general across all types of abuse combined, or for some specific form of abuse). The majority of the studies adopt the health care provider perspective, the hospital perspective or the societal perspective. This is not always clearly stated, but can be inferred from the methods. There are mainly two types of approach to estimate the cost of child maltreatment. 1) The incidence-based approach, which provides an estimate of the total lifetime costs of child maltreatment, including both direct costs (e.g., costs associated with health care and treatment directly related to the maltreatment event) and indirect costs (e.g., costs not directly related to the maltreatment event but as an indirect consequence, as for example productivity losses or costs associated to future substance misuse) resulting from new cases of child maltreatment registered within a defined period of time (generally 1 year). This type of approach requires data on short and long term costs and consequences of child maltreatment such as health status, employment and earnings over lifetime. It is useful for the economic evaluation of prevention programmes

2 The National Longitudinal Survey of Adolescent Health (Add Health) collected data for the purpose of measuring the impact of social environment on adolescent health. It examines the general health and well-being of adolescents in the United States, including1) behaviours that promote health and are detrimental to health; and (2) the influence of community level factors. Dependent variables include diet and nutrition, eating disorders, depression, violent behaviour, intentional injury, unintentional injury, suicide, exercise, health service use, and health insurance coverage. Add Health is a longitudinal study, with adolescents repeatedly interviewed at different time points. For wave III, the third follow-up point, participants were interviewed between August 2001 and April 2002 when they were between 18 and 26 years of age. 
to estimate the potential lifetime cost savings.

2) The prevalence-based approach, which provides an estimate of the total cost (direct and indirect) incurred in a period (generally one year) as a result of the prevalence of child maltreatment during that period, regardless of when the abuse/maltreatment occurred. The studies included in this review adopt both approaches.

The studies included in this review consider both direct and indirect costs of child maltreatment. The type of costs depends on the perspective adopted, and the type of abuse considered. The following is a list of the costs included across the range of studies reviewed: (a) Short and long term health care costs for physical and mental health problems, including depression, post-traumatic stress disorder, self-harm, head injuries, ambulance transport, hospitalisation, emergency admissions, inpatients admissions, transfer to other hospitals, hospital readmissions, hospital rehabilitation, or other medical expenses; (b) Costs associated with alcohol and drug misuse; (c) Productivity losses due to sickness, absenteeism, from unemployment and reduced earnings as a result of being a victim of child abuse; (d) Tax losses due to unemployment; (e) Child social welfare services costs for children living with foster parents, in foster homes, infant homes, in mother support centres, in short-term therapeutic facilities for emotionally disturbed children, and in children's self-reliance support facilities; ( $f$ ) Criminal justice system costs incurred by both the perpetrators of child abuse and subsequently by victims; (h) Special education costs; (i) a range of administrative costs including child consultation centre costs, local government costs, and other administrative expenses; (j) Death related costs, including ambulance transport, coroner/medical examiner costs, suicide, productivity losses due to suicide; (k) Costs due to divorce. Prevalence-based studies typically refer to a one year horizon; incidence-based studies usually consider a lifetime time period. Most studies evaluate the impact of abuse occurring in the age range of 0 to 18 years.
Depending on the approach, incidence and/or prevalence data are derived from setting-specific databases to estimate the affected number of children. Costs are calculated for each item using unit cost data assuming an average consumption of resources per victim. Assumptions may vary according to the specific country, the type of abuse, the expected working age, or expected lifetime. Very few incidence-based studies state if future costs have been discounted and if so which discount rate has been used. ${ }^{3}$ Most studies do not report sensitivity analyses to check the robustness of their results. Sometimes best and worst case scenarios, or a minimum, central and maximum values are reported.

There are very few studies that analyse the cost of child maltreatment in the UK. One study conducted in the UK Royal Liverpool Children's Hospital (Summers, 1992) estimates the financial implications of child maltreatment from the hospital perspective. However, the study refers to children hospitalised in 1990, therefore the results are out of date and take a narrow cost perspective. More recent studies (NSPCC, 2011) review the existing literature covering the prevalence of different types of maltreatment and the impact of maltreatment. However, there is little evidence on costs, either in terms of consequences or interventions to prevent maltreatment. An evaluation of costs in the UK has been performed to assess the consequences of child sexual abuse (Saied-Tessier, 2014). After a review of the literature, the study estimates the cost of sex abuse in the UK using a prevalence approach. There are no UK-based cost studies taking an incidence approach to estimate lifetime costs. There are several incidence-based studies from the USA, Asia, New Zealand, most notably the series of papers by Fang et al., but epidemiological data, socioeconomic conditions and costs structure are very different from the UK. Therefore the results of those studies cannot be generalised to the UK setting.

In conclusion, there are very few studies conducted in the UK that estimate the cost of child maltreatment, and no incidence-based studies of lifetime costs.

3 Discounting is the process of determining the present value of a stream of costs occurring in the future. Given the time value of money, one pound is usually considered to be worth more today than it would be tomorrow. The difference in value between the future and the present is created by discounting the future back to the present using a discount factor, which is a function of time and interest rates. 


\section{Datasets and definition of child maltreatment}

In this section we review the datasets that we considered to estimate the effects of child maltreatment, the definition of $\mathrm{CM}$ adopted in each of them, and their limitations. The datasets and variables used in the analysis are summarised in brief in the Appendix. Each of the datasets used is designed to be nationally representative of the population sampled, though a potential limitation of our analyses is the reduced number of responses to the child maltreatment questions, which may mean the responses are not nationally representative.

\subsection{National Child Development Study}

The first dataset we considered was the National Child Development Study (NCDS). The NCDS follows the lives of over 17,000 people born in England, Scotland and Wales in a single week of 1958. As indicated above, it is the dataset which has been mostly used in the UK literature to examine the effects of CM.

The NCDS includes retrospective measures of $\mathrm{CM}$, which were asked in the biomedical sweep to approximately 9,400 cohort members when they were aged $44 / 45$. We have used the following definitions, following the literature and after consultation with the Advisory Group convened by the NSPCC for this study. ${ }^{4}$

Neglect: an individual is defined as having been neglected in childhood if he/she reports that any of the three following conditions is true, as compared to none of them being true. ${ }^{5}$

1. "Mother (or mother figure) a little or not at all affectionate towards me up to age 16" [Variable CHAD4].

2. "Father (or father figure) a little or not at all affectionate towards me up to age 16" [Variable CHAD1].

3. "I was neglected up to age 16" [Variable CHAD9C].
The prevalence of these three indicators is reported in the tables below.

Table 1: Mother Neglect in NCDS

\begin{tabular}{lcr}
\hline \multicolumn{3}{l}{ Mother (figure) was affectionate towards me } \\
\hline A lot & Freq. & Percent \\
\hline Somewhat & 3,081 & $33.1 \%$ \\
\hline A little & 2,405 & $25.8 \%$ \\
\hline Not at all & 2,563 & $27.5 \%$ \\
\hline I had no mother figure & 851 & $9.1 \%$ \\
\hline Can't say & 253 & $2.7 \%$ \\
\hline Total & 158 & $1.7 \%$ \\
\hline
\end{tabular}

Table 2: Father Neglect in NCDS

\begin{tabular}{lcc}
\hline \multicolumn{3}{l}{ Father (figure) was affectionate towards me } \\
\hline A lot & Freq. & Percent \\
\hline Somewhat & 5,131 & $55.11 \%$ \\
\hline A little & 2,437 & $26.2 \%$ \\
\hline Not at all & 1,287 & $13.8 \%$ \\
\hline I had no father figure & 327 & $3.5 \%$ \\
\hline Can't say & 59 & $0.6 \%$ \\
\hline Total & 70 & $0.8 \%$ \\
\hline
\end{tabular}

Table 3: Neglect in NCDS

\begin{tabular}{lrr}
\hline I was neglected & & \\
\hline & Freq. & Percent \\
\hline Yes & 230 & $2.5 \%$ \\
\hline No & 8,859 & $95.2 \%$ \\
\hline Can't say & 220 & $2.4 \%$ \\
\hline Total & 9,309 & $100.0 \%$ \\
\hline
\end{tabular}

4 Hence, it has been decided not to use the various proxy measures for child maltreatment derived from the childhood years which have been used in the literature, as too aspecific.

5 A separate variable has been included as control in all the estimated models in case the answers include both "I had no mother/ father figure" and "can't say". 
Emotional Abuse: an individual is defined as having been emotionally abused in childhood if he/she reports that any of the three following conditions is true, as compared to none of them being true. ${ }^{6}$

1. "I was verbally abused by a parent (or parent figure) up to age 16" [Variable CHAD9F].

2. "I suffered humiliation, ridicule, bullying or mental cruelty from a parent (or parent figure) up to age 16" [CHAD9G].

3. "I witnessed physical or sexual abuse of others in my family up to age 16" [CHAD9H].

The prevalence of these three indicators is reported in the tables below.

Table 4: Verbal abuse in NCDS

\begin{tabular}{lrr}
\hline \multicolumn{3}{l}{ I was verbally abused by a parent } \\
\hline \multicolumn{3}{l}{ (figure) } \\
\hline Yes & Freq. & Percent \\
\hline No & 746 & $8.0 \%$ \\
\hline Can't say & 8,388 & $90.1 \%$ \\
\hline Total & 175 & $1.9 \%$ \\
\hline
\end{tabular}

Table 5: Suffering humiliation in NCDS

I suffered humiliation, ridicule, bullying or mental cruelty from a parent (figure)

\begin{tabular}{lrr}
\hline & Freq. & Percent \\
\hline Yes & 660 & $7.1 \%$ \\
\hline No & 8,479 & $91.1 \%$ \\
\hline Can't say & 170 & $1.8 \%$ \\
\hline Total & 9,309 & $100.0 \%$ \\
\hline
\end{tabular}

Table 6: Witnessed abuse in NCDS

\begin{tabular}{lrr}
\hline \multicolumn{3}{l}{ I witnessed physical or sexual abuse of others in my family } \\
\hline Yes & Freq. & Percent \\
\hline No & 559 & $6.0 \%$ \\
\hline Can't say & 8,683 & $93.3 \%$ \\
\hline Total & 67 & $0.7 \%$ \\
\hline
\end{tabular}

Physical Abuse: an individual is defined as having been physically abused in childhood if he/she reports yes to "I was physically abused by a parent - punched, kicked or hit or beaten with an object, or needed medical treatment up to age 16" [Variable CHAD9I]. ${ }^{7}$ The prevalence of this indicator is reported in the table below.

Table 7: Physical abuse in NCDS

\begin{tabular}{lcr}
\hline \multicolumn{3}{l}{ I was physically abused by a parent (figure) } \\
\hline Yes & Freq. & Percent \\
\hline No & 559 & $6.0 \%$ \\
\hline Can't say & 8,663 & $93.1 \%$ \\
\hline Total & 87 & $0.9 \%$ \\
\hline
\end{tabular}

Sexual Abuse: an individual is defined as having been sexually abused in childhood if he/she reports yes to "I was sexually abused by a parent (or parentfigure)" [Variable CHAD9K]. ${ }^{8}$ The prevalence of this indicator is reported in the table below.

Table 8: Sexual abuse in NCDS

\begin{tabular}{lrr}
\hline \multicolumn{4}{l}{ I was sexually abused by a parent (figure) } \\
\hline Yes & Freq. & Percent \\
\hline No & 143 & $1.5 \%$ \\
\hline Can't say & 9,124 & $98.0 \%$ \\
\hline Total & 42 & $0.5 \%$ \\
\hline
\end{tabular}

Additionally, in order to develop estimates encompassing an overall effect of CM, we constructed a "global" measure, where CM is said to have occurred if the individual reports having experienced any of the above forms of CM.

6 A separate variable has been included as control in all the estimated models in case the answers include both "no" and "can't say".

7 A separate variable has been included as control in all the estimated models in case the answer is "can't say".

8 According to the Denholm et al sexual abuse is defined as "any completed or attempted sexual act, sexual contact or non-contact sexual interaction with a child by a caregiver". 


\subsection{English Longitudinal Study of Ageing}

The second dataset we considered was the English Longitudinal Study of Ageing (ELSA). This is a longitudinal survey of ageing and quality of life among people aged 50+, which began in 2002.

The ELSA includes retrospective measures of CM (physical abuse and neglect only), which were asked in the life history module in wave $3 .^{9}$ We have used the following definitions. ${ }^{10}$

Neglect: an individual is defined as having been neglected in childhood if he/she answers "agree or strongly agree" to the question "Mother (mother figure) or Father (father figure) seemed emotionally cold to me". The prevalence of these two indicators is reported in the tables below.

Table 9: Mother Neglect in ELSA

\begin{tabular}{lcc}
\hline \multicolumn{3}{l}{ Mother (figure) seemed emotionally cold to me } \\
\hline Strongly agree & Freq. & Percent \\
\hline Agree & 207 & $3.3 \%$ \\
\hline Disagree & 660 & $10.5 \%$ \\
\hline Strongly disagree & 1,969 & $31.2 \%$ \\
\hline Total & 3,475 & $55.1 \%$ \\
\hline
\end{tabular}

Table 10: Father Neglect in ELSA

\begin{tabular}{lcc}
\hline \multicolumn{3}{l}{ Father (figure) seemed emotionally cold to me } \\
\hline Strongly agree & Freq. & Percent \\
\hline Agree & 236 & $3.9 \%$ \\
\hline Disagree & 743 & $12.1 \%$ \\
\hline Strongly disagree & 2,296 & $37.4 \%$ \\
\hline Total & 2,862 & $46.7 \%$ \\
\hline
\end{tabular}

Physical Abuse: an individual is defined as having been physically abused in childhood if he/she answers yes to the question "When you were aged under 16, were you physically abused by your parents". The prevalence of this indicator is reported in the table below.

Table 11: Physical Abuse in ELSA

\begin{tabular}{lcc}
\hline \multicolumn{3}{l}{ Whether when aged $<16$ was physically abused by your parents } \\
\hline & Freq. & Percent \\
\hline Yes & 227 & $3.5 \%$ \\
\hline No & 6,290 & $96.5 \%$ \\
\hline Total & 6,517 & $100.0 \%$ \\
\hline
\end{tabular}

Additionally, also for the ELSA we have constructed a "global" measure, which is said to occur if the individual reports to have experienced any of the above forms of CM.

\subsection{Edinburgh Study of Youth Transitions and Crime}

The third dataset we considered was the Edinburgh Study of Youth Transitions and Crime (ESYTC). This is a longitudinal (4 waves) study of around 4,300 11-12 year olds in Edinburgh in 1998.

The ESYTC is the only dataset which includes unique information (in waves 3 and 4) from the Social Work Monitoring Form and the Children's Hearing Monitoring Form as to whether the child was referred to the social work department or to the children's reporter for "specific issues raised" related to sexual and physical abuse and for "issues at home" related to neglect by parents. Unfortunately, this information proved not to be useful. While a substantial number of respondents had either a social work record (78\% [n=357] in wave 3 and $9.2 \%$ $[n=422]$ in wave 4 ) or a children's hearing record (6.4\% [ $n=293]$ in wave 3 and $8.9 \%$ [ $n=407]$ in wave 4), among these those reporting cases of physical and sexual abuse amounted only to a number of cases between 6 and 12 - hence the numbers were too small to perform any meaningful analysis. ${ }^{11}$

9 The ELSA wave 3 is a four year follow up of the English population aged 50 an over at the first wave started in 2002.

10 No paper using these variables from the ELSA has been published, to the best of our knowledge.

11 It is possible that this low prevalence arises from the fact that only the main reason for referral is reported in the forms. 


\subsection{British Household Panel Study/ UK Household Longitudinal Study}

The last two datasets we considered were the British Household Panel Study (BHPS) and the UK Household Longitudinal Study (UKHLS). The BHPS began in 1991 with more than 5,000 households, and 10,300 individuals drawn from 250 areas of Great Britain. Additional samples of 1,500 households in each of Scotland and Wales were added to the main sample in 1999, and in 2001 a sample of 2,000 households was added in Northern Ireland, making the panel suitable for UKwide research. The BHPS surveyed people annually until 2008, for 18 waves. In 2008, 6,700 of the over 8,000 BHPS participants accepted to participate in Understanding Society, which started in 2009 and follows the lives of over 40,000 households.

While there is no direct information on CM asked in the BHPS or in the UKHLS, we had identified two types of proxy information.

1. Parenting questions similar to those used in the economics literature based on the AddHealth data, available in the BHPS waves 12, 13 and 17. These questions were asked to the parents of all children aged 0 to 15 at each of these three waves.

a. How often do you spank or slap your child/any of your children?

b. How often do you hug or cuddle your child/any of your children?

c. How often do you shout at your child/any of your children?

With possible answers: never/seldom/ sometimes/very often.

Upon consultation with the Advisory Group, the following indicators have been constructed.
Neglect: a child is defined as neglected if the parents report they hug or cuddle him/her "never or seldom". The prevalence of this indicator is reported in the table below.

Table 12: Neglect in BHPS

\begin{tabular}{|c|c|c|c|}
\hline \multicolumn{4}{|c|}{ How often do you cuddle or hug <NAME>? } \\
\hline & Wave 12 & Wave 13 & Wave 17 \\
\hline Never & $3.1(201)$ & $4.1(67)$ & $2.1(138)$ \\
\hline Seldom & $4.0(256)$ & $6.1(99)$ & $4.0(261)$ \\
\hline Sometimes & $15.6(1,003)$ & $19.7(322)$ & $15.4(1,011)$ \\
\hline Very often & $77.3(4,957)$ & $70.2(1,147)$ & $78.5(5,138)$ \\
\hline Total & $100.0(6,417)$ & $100.0(1,635)$ & $100.0(6,548)$ \\
\hline
\end{tabular}

Note: the numbers in each cell are the percentages, in parentheses the frequencies.

Emotional abuse: a child is defined as emotionally abused if the parents reply they yell at him/her "very often". The prevalence of this indicator is reported in the table below. The table reveals a prevalence of emotional abuse ranging between $21.9 \%$ (wave 17) and $29.1 \%$ (wave 12), which is extremely high as compared to ranges reported elsewhere: for example, the figure reported in the report "Abuse and Neglect in the UK today" 12 is roughly a third of this value, i.e. $7 \%$. It is unclear whether this discrepancy reflects a problem with the responses in the "Abuse and Neglect in the UK today" report or the BHPS responses, or is a genuine difference; assuming the data from the "Abuse and Neglect in the UK today" report are correct, they cast doubts on the measure used in our analysis.

Table 13: Emotional Abuse in BHPS

\begin{tabular}{lccc}
\hline \multicolumn{4}{l}{ And how often do you yell at <NAME>? } \\
\hline Never & Wave 12 & Wave 13 & Wave 17 \\
\hline Seldom & $11.2(716)$ & $13.8(225)$ & $13.6(887)$ \\
\hline Sometimes & 42.0 (2,697) & $40.4(660)$ & $42.9(2,812)$ \\
\hline Very often & $29.1(1,869)$ & $24.0(393)$ & $21.9(1,434)$ \\
\hline Total & $100.0(6,415)$ & $100.0(1,635)$ & $100.0(6,547)$ \\
\hline
\end{tabular}

Note: the numbers in each cell are the percentages, in parentheses the frequencies. 
Physical abuse: a child is defined as physically abused if the parents reply they spank or slap him/ her "very often", since this definition aligns nicely with the one used in the NSPCC questionnaire "National Survey of Child Safety and Victimisation". ${ }^{13}$ The prevalence of this indicator is reported in the table below. The table reveals that the reported prevalence of physical abuse in the BHPS is extremely low, perhaps due to the self-reported nature of the survey which might prevent parents from reporting certain types of behaviours.

Table 14: Physical Abuse in BHPS

\begin{tabular}{lccc}
\hline \multicolumn{4}{l}{ And how often do you spank or slap <NAME>? } \\
\hline Never & Wave 12 & Wave 13 & Wave 17 \\
\hline Seldom & $63.8(4,096)$ & $60.9(996)$ & $73.6(4,820)$ \\
\hline Sometimes & $24.3(1,560)$ & $26.8(438)$ & $18.7(1,225)$ \\
\hline Very often & $10.5(674)$ & $10.4(170)$ & $6.6(435)$ \\
\hline Total & $100,0(6,417)$ & $1.8(30)$ & $1.0(67)$ \\
\hline
\end{tabular}

Note: the numbers in each cell are the percentages, in parentheses the frequencies.

2. Module on Parenting Styles asked in waves 3, 4 and 5 of the UKHLS, derived from the Parenting Styles and Dimensions Questionnaire. ${ }^{14}$ Upon consultation with the Advisory Group, the following indicators have been constructed.

Neglect: a child is defined as neglected if the parents report "never/once in a while/about half the time responsive to the child's feelings and needs", as opposed to "very often/always" (item 1 of the PSDQ).
Emotional abuse: a child is defined as emotionally abused if the parents reply "always/very often" to either of the following three items - "yell or shout when child misbehaves" (item 13); "explode in anger towards child" (item 16); "punish child by putting him/her somewhere alone with little or no explanation" (item 28).

Physical abuse: a child is defined as physically abused if the parents reply "always/very often" to either of the following three items - "use physical punishment as a way of disciplining child" (item 2); "spank child when he/she is disobedient" (item 6); "slap child when he/she misbehaves" (item 32).

Unfortunately, this module was designed in such a way that the questions were only asked about 10-year old children. Hence, this precluded the use of methods which exploit variation within siblings, which is the main advantage of the UKHLS. Additionally, the prevalence rates obtained from this survey were somewhat inconsistent with other sources: while the prevalence of neglect (as defined above) ranged between 8-9\%, the prevalence of emotional abuse was as high as $21-23 \%$ using the first definition ("yell or shout"), but as low as 1-2\% when using the other two; likewise, the prevalence of physical abuse ranged between $0.50-2 \%$, effectively not yielding a sufficient sample size to carry out meaningful analysis. This casts some doubts on the usefulness of the Parenting Styles and Dimensions Questionnaire to construct child maltreatment indicators. For all these considerations, we have decided not to perform the analysis of the effects of CM based on the UKHLS data.

13 Radford L., Corral S., Bradley C., Fisher H et al. (2011) “Child abuse and neglect in the UK today”. NSPCC London. Appendix A. pg. 124-137

14 Robinson CC, Mandleco B, Oslen SF, Hart CH. The Parenting Styles and Dimensions Questionnaire (PSQD). Instruments and Index. In: Perlmutter BF, Touliatos J, Holden GW, editors. Thousand Oaks: Sage, 2001: 319-321 


\section{The effects of child maltreatment}

The key challenge in estimating the effects of child maltreatment on different health and socioeconomic outcomes arises from the fact that parents maltreating their children are not a random sample of the population, hence care needs to be taken in disentangling the effect of CM from that of other characteristics associated with these families (e.g. poverty or other adversities).

Given the data limitations noted in the section above, we are left with only two datasets with retrospective information to estimate the effects of CM: the NCDS and the ELSA. ${ }^{15}$ Both datasets only follow individuals; hence we cannot rely on any within-family analysis as done in the US economic literature. Our approach therefore consists in exploiting the richness of both datasets and to account progressively in the estimated regressions for a rich set of background characteristics, predetermined with respect to $\mathrm{CM}$, and to see how the estimated coefficients change when accounting for them. It should be borne in mind that both datasets report retrospective measures of child maltreatment; these rely on respondent recall, which may be imperfect. Also, these measures preclude cases of child maltreatment that resulted in the death of the child. Given the key challenge highlighted above, while we adjusted for a range of potential confounding factors, we are unable to say for certain whether maltreatment directly impacted on the outcomes considered or was spuriously associated with them. Reverse causality or bidirectional relationships can also not be ruled out for some of the associations found, especially those between maltreatment and potentially chronic health problems.
The following outcomes were selected for analysis, on the basis of the findings from the literature review, and to inform the subsequent cost analysis.

1) Physical Health Problems: ${ }^{16}$
a. Obesity
b. Hypertension
c. Diabetes
d. Cancer

2) Mental Health Problems (diagnosis)
a. Any type
b. Anxiety
C. Depression

3) Healthy Behaviours
a. Heavy Drinking (consuming 2 or more alcoholic drinks a day)
b. Smoking
c. Heavy Smoking (25 cigarettes per day or more)

4) Labour Market Outcomes
a. Employment
b. Net Weekly Earnings (if Employed)
c. Gross Weekly Earnings (if Employed)

5) Welfare Use

a. Weekly Disability-Related Benefits

15 We also performed analyses of the effects of neglect and physical abuse on the basis of the BHPS data. For doing so, we used the CM indicators constructed on the basis of the information reported in waves 12, 13 and 17 of the BHPS, and measured the outcomes in the UKHLS waves 2 to 5, exploiting the fact that the BHPS sample had been incorporated in the UKHLS at wave 2. Given that wave 12 of the BHPS occurred in 2002 and wave 5 of the USOC (Understanding Society) in 2014, and that an individual has to be at least 16 years old to be interviewed in the main interview, the youngest child in our sample is 16 in 2014 , when we can measure the outcomes, i.e. he/she is born in 1998 and is 4 years old in the wave 12 of the BHPS, when we can measure maltreatment. On the other hand, the older child in our sample is 15 at wave 12 of the BHPS (the latest age at which the maltreatment questions are asked to the parents), so that he/she is 27 at wave 5 of the USOC (when we can measure the outcomes). Unfortunately, such analyses led to inconclusive results, probably due to the limited variation in the sample and the nature of the $\mathrm{CM}$ variables, derived from parenting questions asked within a household survey.

16 It was decided not to use objective measures, i.e. those based on biomarkers, since individuals in that case might not be aware of the condition and so might not bear the corresponding costs. 


\subsection{National Child Development Study}

The NCDS results are reported in Tables 15-19. For Tables 15-18, each table presents results for a set of outcomes, according to the domains outlined in the previous section; Table 19 presents the results for the global measure of CM. For each outcome, each row reports the results for a particular sweep, where the effective number of sweeps used depends on the availability and the prevalence of a certain outcome. ${ }^{17}$ For Tables $15-18$, each table further comprises 12 columns, 4 for each measure of CM; for Table 19, we present 4 columns for each outcome. In either case, each of the 4 columns reports the regression coefficients from a particular specification, where we add incrementally more controls for background conditions and other adverse early experiences. ${ }^{18}$

Table 15 shows that we are unable to detect robust effects of CM on our measures of physical health. The only exception occurs in the case of physical abuse and obesity: individuals who have been physically abused in their childhood are between 4 and 7 percentage points more likely to be obese in their 40s and 50s; the estimated coefficients are barely affected by the inclusion of an extended set of controls.

Table 16, instead, shows that CM has persistent, sizeable and significant effects for mental health, with the more negative consequences being suffered by those individuals who experienced sexual abuse. Although the magnitude of the estimated coefficients is roughly halved upon the inclusion of the extended set of controls (columns (4)), the effects are persistent, and range from 1.4 percentage point increase in the probability of being diagnosed for anxiety in the case of neglect to a sizeable 16.5 percentage point increase in the case of sexual abuse.
The effects on healthy behaviours are reported in Table 17. We are unable to detect any impact of CM on heavy drinking or heavy smoking, while we find a significant and sizeable effect of the three forms of abuse on the probability of being a current smoker; such effects are stronger in the case of sexual abuse, however they seem to diminish over time.

Lastly, Table 18 shows that there is very little effect of CM on labour market outcomes: the only effect we are able to detect is a reduction in the probability of employment (of a modest magnitude between 3-5 percentage points) among those who have experienced physical abuse before age 16.

Turning to the estimates for the global measure of CM, Table 19 shows no significant impact on physical health: the association with obesity detected for physical abuse disappears when a more encompassing measure is adopted. Instead, $\mathrm{CM}$ in any form continues to have a strong and sizeable impact on mental health, ranging from an increase by 2.6 percentage points for anxiety at 33 years to an increase by 8.5 percentage points for any mental health problem at 42 years. The results obtained from using the global measure of CM are the same as those seen when using the more disaggregated measure: the only significant and robust finding is an increase of the order of 3.23.4 percentage points of the probability of being a current smoker, which nonetheless fades out by age 55. Lastly, we detect a negative and significant association between having experienced any CM and the probability of being in paid employment at ages 42 and 50, by 2.7-3.6 percentage points.

To inform our cost analysis (see below) we reran the analysis using selected outcomes from Sweep 4 of the NCDS (age 23). The results are in the appendix (Table A2). 


\subsection{English Longitudinal Study of Ageing}

The ELSA results are reported in Tables 20-23. Here we only have two indicators of $\mathrm{CM}$, namely neglect and physical abuse (Tables 20-21), which we also combine in the global measure of any form of CM (Tables 22-23). For both of these indicators, we report the results from two specifications (columns 1 and 2): the first one includes a basic set of demographics, and the second one adds a more extended set of controls. ${ }^{19}$

The ELSA results are remarkably consistent with the NCDS results. The only robust effect of $\mathrm{CM}$ on physical health is an increase in the probability of being obese, which is estimated as 8 percentage points in the case of physical abuse (Table 20). On the other hand, both indicators of $\mathrm{CM}$ are significantly and robustly associated with all the measures of mental health (Table 20). Instead, we find opposite effects on heavy drinking: while having experienced neglect is positively associated with it, we detect a negative association of having experienced physical abuse. Then, no impact of neglect is detected on smoking, while having experienced physical abuse is associated with an increase in the probability of being a smoker by 6 percentage points (Table 21). Again, as for the NCDS little impact on labour market outcomes is detected. Lastly, we find a sizeable and significant effect of CM on the probability of receiving welfare: the amount of weekly disability-related benefits is higher by $£ 31$ per week for individuals who have experienced neglect, and by $£ 111$ per week for those who report to have experienced physical abuse (Table 21). When we use the global measure, just as seen for the NCDS, the significant effect of physical abuse on obesity disappears, while the effect of having experienced any CM on mental health remains sizeable and strongly significant. Lastly, among the health behaviours, we find a strong and significant impact of any CM on heavy drinking (2.9 percentage points), and on receipt of disability benefits ( $£ 35.59$ GBP per week), but not on other labour market outcomes (Table 22).

In sum, having experienced any CM in childhood is associated with worse mental health outcomes, smoking behaviour, alcohol use, lower probability of employment and greater welfare dependence; no robust effects are detected for physical health, heavy smoking and wages. 


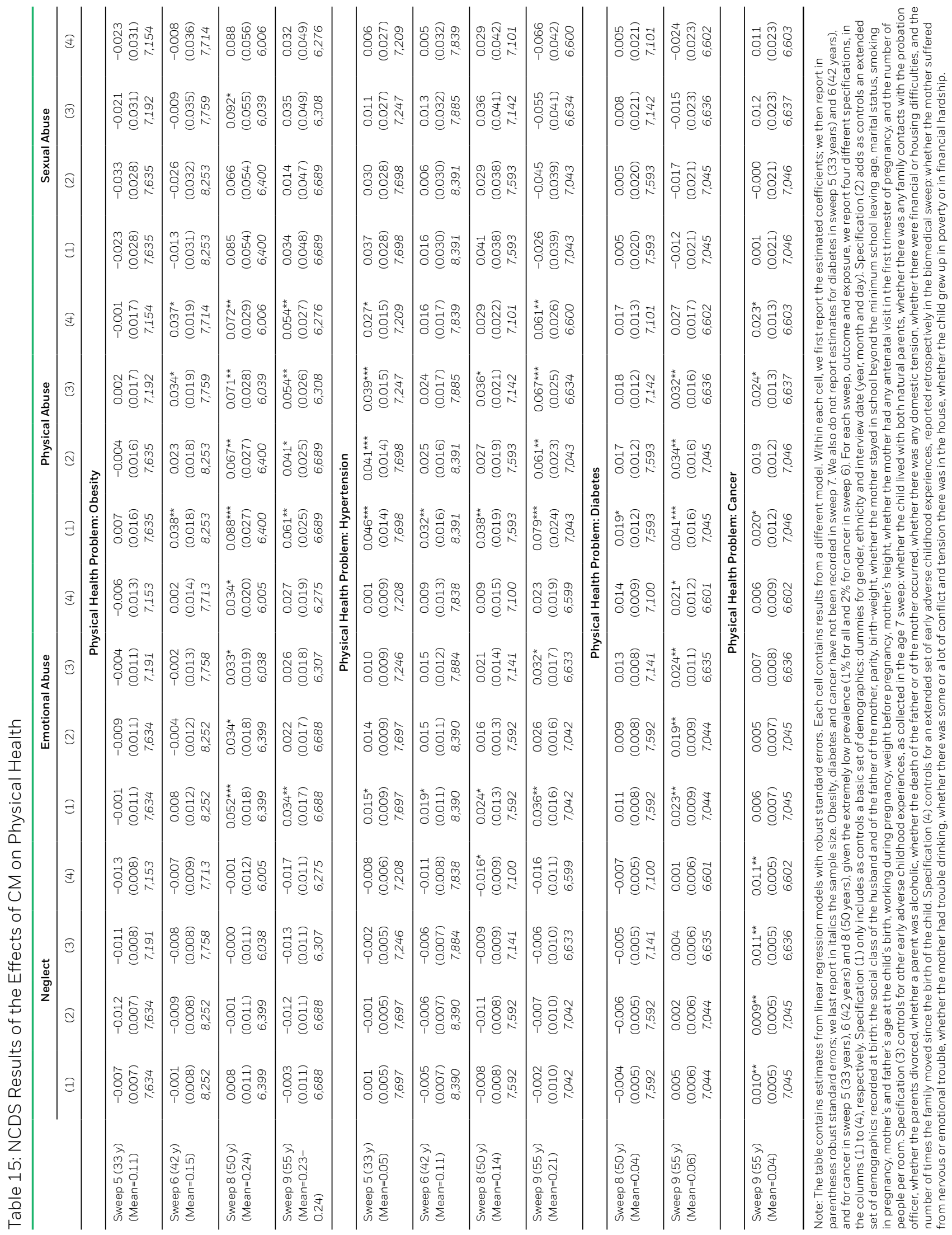




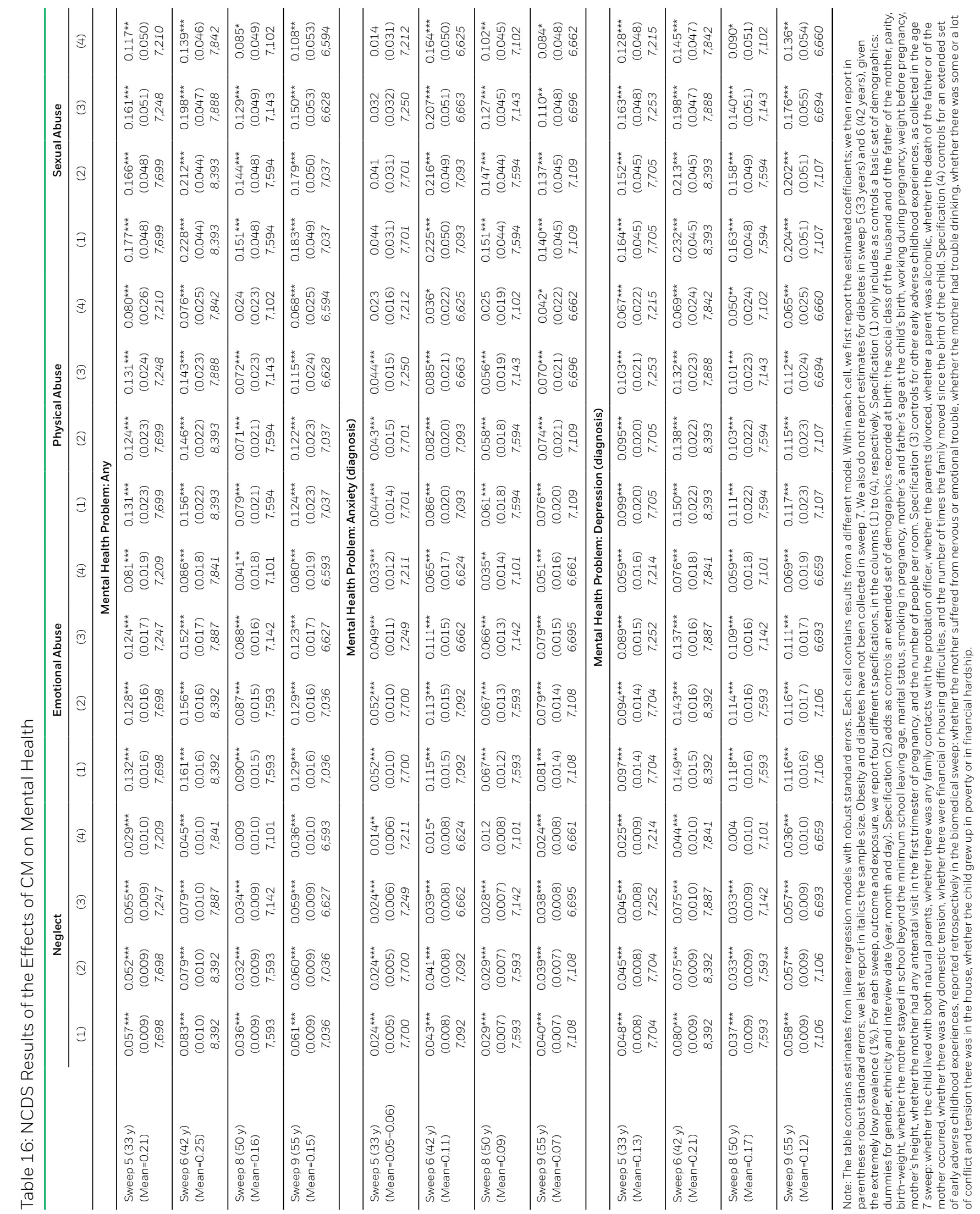




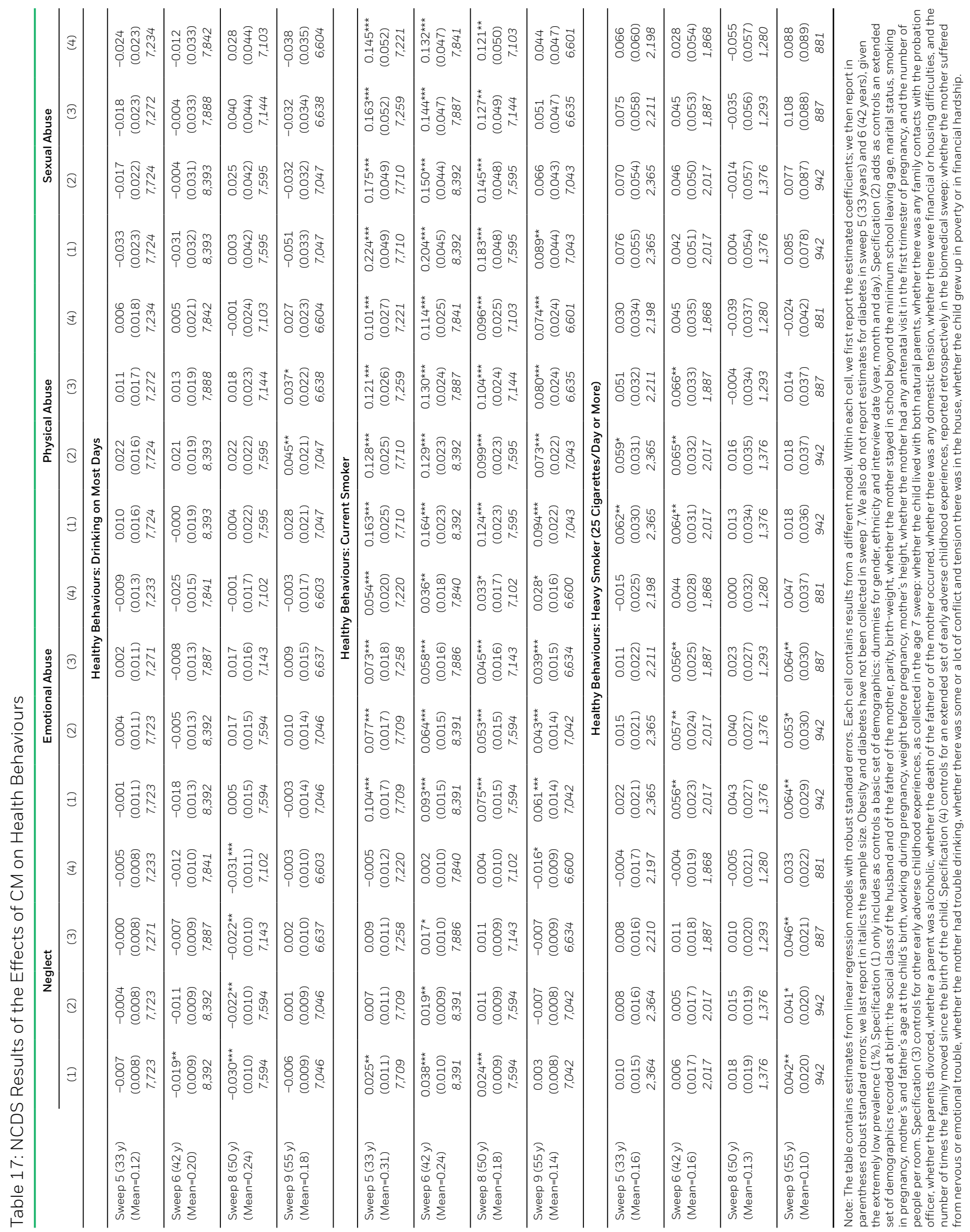




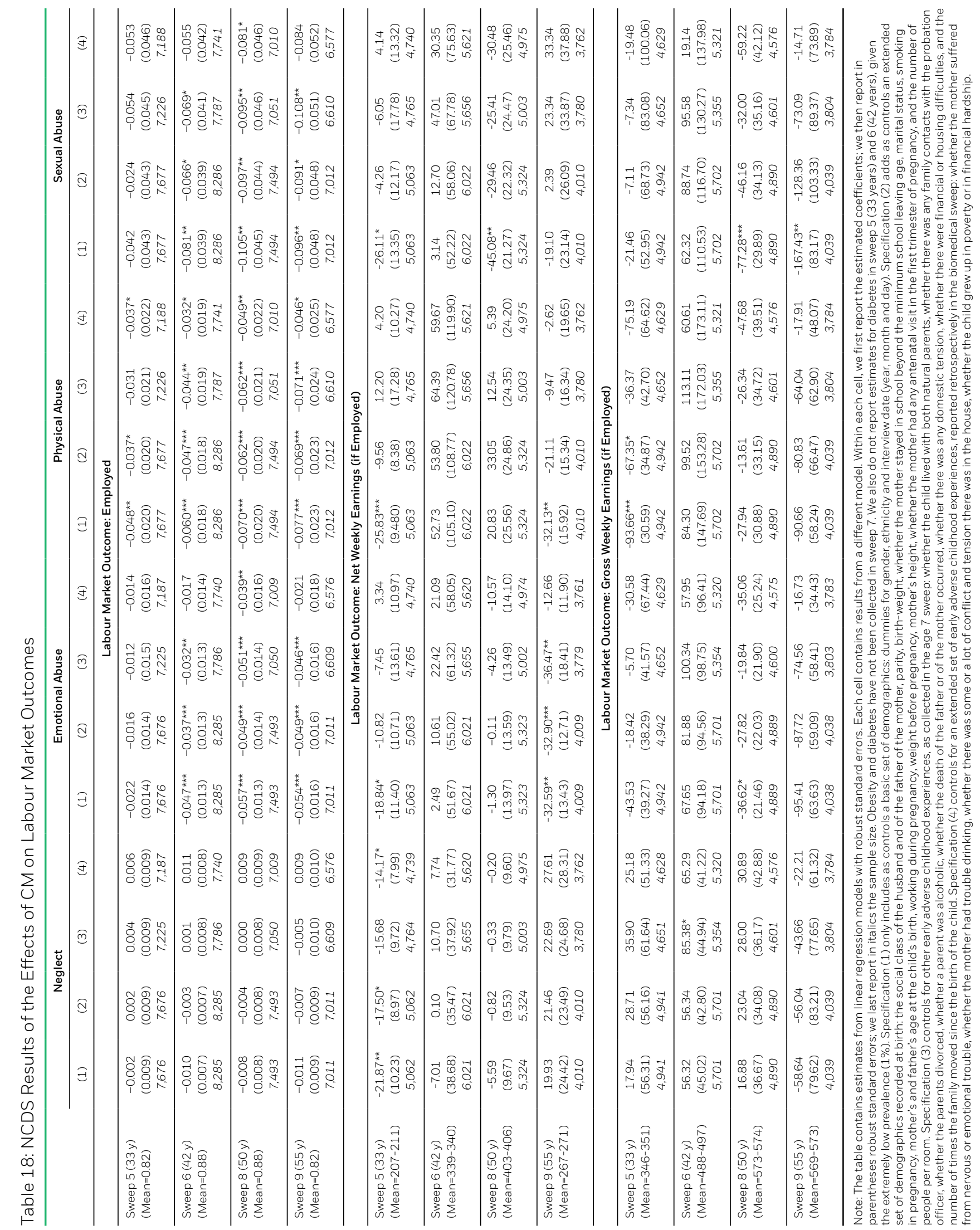




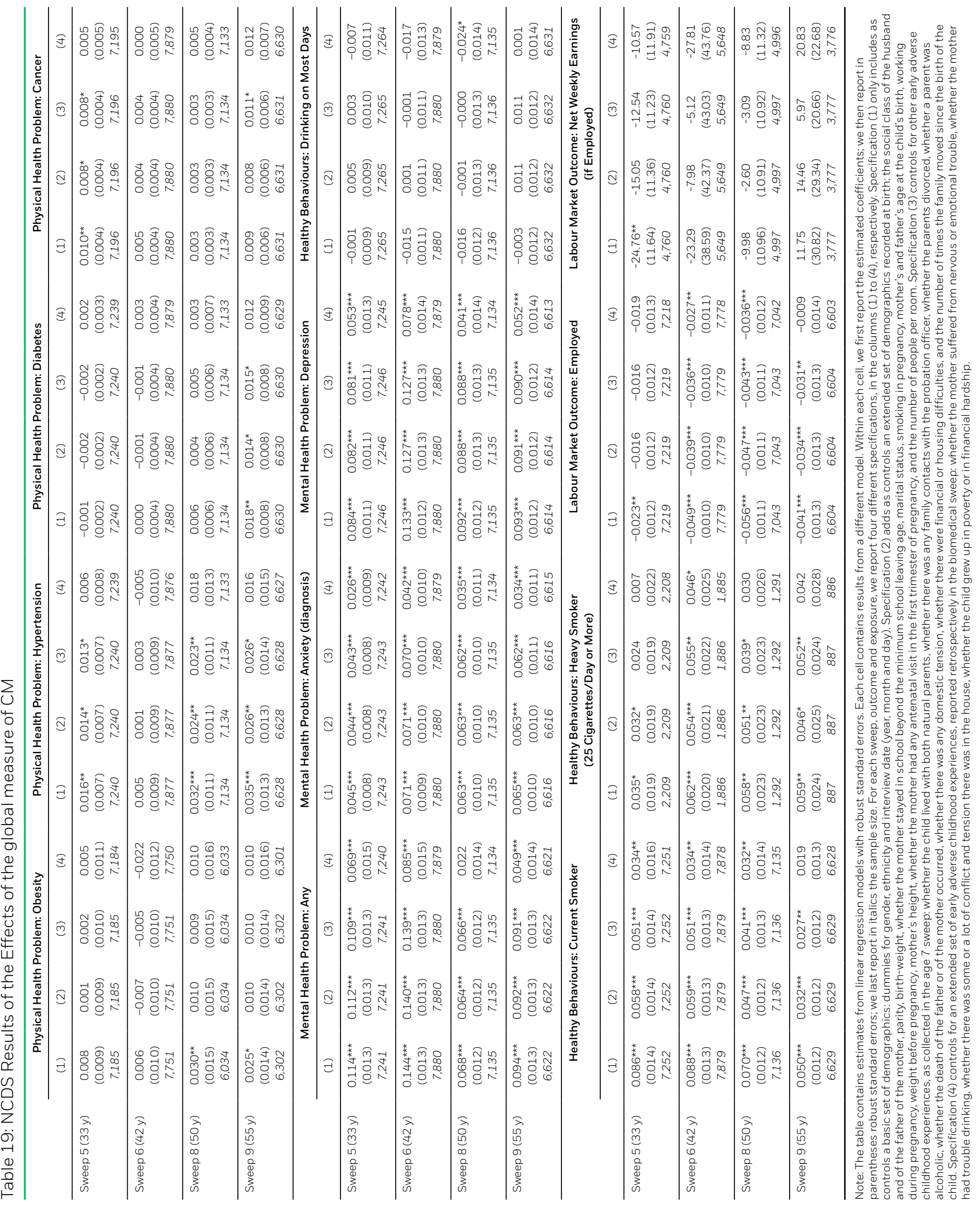


Table 20: ELSA Results of the Effects of CM on Physical and Mental Health

\begin{tabular}{|c|c|c|c|c|}
\hline & \multicolumn{2}{|c|}{ Neglect } & \multicolumn{2}{|c|}{ Physical Abuse } \\
\hline & $(1)$ & $(2)$ & $(1)$ & $(2)$ \\
\hline \multicolumn{5}{|c|}{ Physical Health Problem: Obesity } \\
\hline $\begin{array}{l}\text { All ages (HSE) } \\
(\text { Mean=0.25) }\end{array}$ & $\begin{array}{c}0.022(0.014) \\
31,131\end{array}$ & $\begin{array}{c}0.025^{\star}(0.015) \\
30,726\end{array}$ & $\begin{array}{c}0.047(0.034) \\
30,818\end{array}$ & $\begin{array}{c}0.044(0.035) \\
30,245\end{array}$ \\
\hline $\begin{array}{l}\text { All ages (nurse) } \\
\text { (Mean }=0.30)\end{array}$ & $\begin{array}{c}0.032^{* *}(0.014) \\
13,358\end{array}$ & $\begin{array}{c}0.026^{\star}(0.015) \\
13,178\end{array}$ & $\begin{array}{c}0.093^{\star \star \star}(0.034) \\
13,232\end{array}$ & $\begin{array}{c}0.080^{* \star}(0.035) \\
13,058\end{array}$ \\
\hline \multicolumn{5}{|c|}{ Physical Health Problem: Hypertension } \\
\hline $\begin{array}{l}\text { All ages } \\
\text { (Mean=0.43-0.44) }\end{array}$ & $\begin{array}{c}0.014(0.013) \\
33,257\end{array}$ & $\begin{array}{c}0.018(0.014) \\
32,824\end{array}$ & $\begin{array}{c}0.047(0.032) \\
32,945\end{array}$ & $\begin{array}{c}0.040(0.033) \\
32,524\end{array}$ \\
\hline \multicolumn{5}{|c|}{ Physical Health Problem: Diabetes } \\
\hline $\begin{array}{l}\text { All ages } \\
(\text { Mean=0.10) }\end{array}$ & $\begin{array}{c}0.007(0.008) \\
33,052\end{array}$ & $\begin{array}{c}0.008(0.009) \\
32,619\end{array}$ & $\begin{array}{c}0.054^{\star \star}(0.022) \\
32,741 \\
\end{array}$ & $\begin{array}{c}0.058^{\star \star \star}(0.022) \\
32,320 \\
\end{array}$ \\
\hline $\begin{array}{l}\text { By age } 50 \\
(\text { Mean }=0.02)\end{array}$ & $\begin{array}{c}0.002(0.004) \\
32,566\end{array}$ & $\begin{array}{c}0.003(0.004) \\
32,144\end{array}$ & $\begin{array}{c}0.004 \text { (0.009) } \\
32,261\end{array}$ & $\begin{array}{c}0.006(0.010) \\
31,851\end{array}$ \\
\hline $\begin{array}{l}\text { By age } 60 \\
(\text { Mean }=0.04)\end{array}$ & $\begin{array}{c}0.005(0.006) \\
32,566\end{array}$ & $\begin{array}{c}0.007(0.007) \\
32,144\end{array}$ & $\begin{array}{c}0.026(0.017) \\
32,261\end{array}$ & $\begin{array}{c}0.029^{*}(0.017) \\
31,851 \\
\end{array}$ \\
\hline $\begin{array}{l}\text { By age } 70 \\
\text { (Mean=0.07) }\end{array}$ & $\begin{array}{c}0.009(0.007) \\
32,566 \\
\end{array}$ & $\begin{array}{c}0.010(0.008) \\
32,144 \\
\end{array}$ & $\begin{array}{c}0.038^{\star \star}(0.019) \\
32,261 \\
\end{array}$ & $\begin{array}{c}0.039 *(0.020) \\
31,851 \\
\end{array}$ \\
\hline \multicolumn{5}{|c|}{ Physical Health Problem: Cancer } \\
\hline $\begin{array}{l}\text { All ages } \\
(\text { Mean=0.08) }\end{array}$ & $\begin{array}{c}-0.001(0.007) \\
33,034\end{array}$ & $\begin{array}{c}-0.002(0.007) \\
32,601\end{array}$ & $\begin{array}{c}0.024(0.017) \\
32,721\end{array}$ & $\begin{array}{c}0.023(0.018) \\
32,300\end{array}$ \\
\hline \multicolumn{5}{|c|}{ Mental Health Problem: Emotional/psychotic disorder (all types) } \\
\hline $\begin{array}{l}\text { All ages } \\
(\text { Mean }=0.10-0.11)\end{array}$ & $\begin{array}{c}0.065^{\star \star \star}(0.010) \\
33,083 \\
\end{array}$ & $\begin{array}{c}0.042^{\star * *}(0.010) \\
32,650\end{array}$ & $\begin{array}{c}0.177^{\star \star \star}(0.029) \\
32,771\end{array}$ & $\begin{array}{c}0.129^{\star \star \star}(0.029) \\
32,350\end{array}$ \\
\hline $\begin{array}{l}\text { By age } 30 \\
(\text { Mean }=0.02)\end{array}$ & $\begin{array}{c}0.012^{\star *}(0.005) \\
32,020 \\
\end{array}$ & $\begin{array}{c}0.008(0.005) \\
31,613 \\
\end{array}$ & $\begin{array}{c}0.037^{\star *}(0.017) \\
31,714\end{array}$ & $\begin{array}{c}0.025(0.017) \\
31,319 \\
\end{array}$ \\
\hline $\begin{array}{l}\text { By age } 40 \\
\text { (Mean=0.04) }\end{array}$ & $\begin{array}{c}0.020^{\star \star \star}(0.006) \\
32,020\end{array}$ & $\begin{array}{c}0.012^{\star}(0.006) \\
31,613\end{array}$ & $\begin{array}{c}0.051^{\star *}(0.020) \\
31,714\end{array}$ & $\begin{array}{c}0.030(0.020) \\
31,319\end{array}$ \\
\hline $\begin{array}{l}\text { By age } 50 \\
\text { (Mean=0.06) }\end{array}$ & $\begin{array}{c}0.037^{\star \star \star \star}(0.008) \\
32,020\end{array}$ & $\begin{array}{c}0.021^{\star \star \star}(0.008) \\
31,613\end{array}$ & $\begin{array}{c}0.130^{\star \star \star}(0.028) \\
31,714\end{array}$ & $\begin{array}{c}0.100^{\star \star \star}(0.027) \\
31,319\end{array}$ \\
\hline $\begin{array}{l}\text { By age } 60 \\
\text { (Mean=0.07) }\end{array}$ & $\begin{array}{c}0.046^{\star \star \star}(0.009) \\
32,020\end{array}$ & $\begin{array}{c}0.029 * \star \star \\
31,613\end{array}$ & $\begin{array}{c}0.140^{\star \star \star}(0.029) \\
31,714\end{array}$ & $\begin{array}{c}0.110^{\star \star \star}(0.029) \\
31,319\end{array}$ \\
\hline $\begin{array}{l}\text { By age } 70 \\
(\text { Mean=0.07) }\end{array}$ & $\begin{array}{c}0.047^{\star \star \star \star}(0.009) \\
32,020\end{array}$ & $\begin{array}{c}0.031^{\star \star \star}(0.009) \\
31,613\end{array}$ & $\begin{array}{c}0.143^{\star \star \star}(0.029) \\
31,714\end{array}$ & $\begin{array}{c}0.112^{\star \star \star}(0.029) \\
31,319\end{array}$ \\
\hline \multicolumn{5}{|c|}{ Mental Health Problem: Anxiety } \\
\hline $\begin{array}{l}\text { All ages } \\
\text { (Mean=0.07) }\end{array}$ & $\begin{array}{c}0.053^{\star \star \star *}(0.008) \\
31,934\end{array}$ & $\begin{array}{c}0.036^{* * \star}(0.008) \\
31,510\end{array}$ & $\begin{array}{c}0.137^{\star \star \star}(0.027) \\
31,629\end{array}$ & $\begin{array}{c}0.096^{\star \star \star}(0.027) \\
31,217\end{array}$ \\
\hline $\begin{array}{l}\text { By age } 30 \\
(\text { Mean }=0.01-0.02)\end{array}$ & $\begin{array}{c}0.009 * \star(0.004) \\
31,209\end{array}$ & $\begin{array}{c}0.007(0.005) \\
30,810\end{array}$ & $\begin{array}{c}0.024^{\star}(0.014) \\
30,906\end{array}$ & $\begin{array}{c}0.015(0.014) \\
30,519\end{array}$ \\
\hline $\begin{array}{l}\text { By age } 40 \\
(\text { Mean=0.02-0.03) }\end{array}$ & $\begin{array}{c}0.015^{\star \star *}(0.005) \\
31,209\end{array}$ & $\begin{array}{c}0.010 *(0.006) \\
30,810 \\
\end{array}$ & $\begin{array}{c}0.038^{\star \star}(0.018) \\
30,906\end{array}$ & $\begin{array}{c}0.023(0.018) \\
30,519\end{array}$ \\
\hline $\begin{array}{l}\text { By age } 50 \\
(\text { Mean=0.04) }\end{array}$ & $\begin{array}{c}0.029^{\star \star \star}(0.007) \\
31,209\end{array}$ & $\begin{array}{c}0.018^{\star \star \star}(0.007) \\
30,810\end{array}$ & $\begin{array}{c}0.097^{\star \star \star}(0.025) \\
30,906\end{array}$ & $\begin{array}{c}0.075^{\star \star \star}(0.025) \\
30,519\end{array}$ \\
\hline $\begin{array}{l}\text { By age } 60 \\
\text { (Mean=0.05) }\end{array}$ & $\begin{array}{c}0.035^{\star \star \star}(0.007) \\
31,209 \\
\end{array}$ & $\begin{array}{c}0.023^{* * *}(0.008) \\
30,810 \\
\end{array}$ & $\begin{array}{c}0.093^{\star \star \star}(0.026) \\
30,906 \\
\end{array}$ & $\begin{array}{c}0.068^{\star \star \star}(0.025) \\
30,519 \\
\end{array}$ \\
\hline $\begin{array}{l}\text { By age } 70 \\
(\text { Mean=0.05) }\end{array}$ & $\begin{array}{c}0.036^{\star \star \star}(0.007) \\
31,209\end{array}$ & $\begin{array}{c}0.024^{* \star *}(0.008) \\
30,810\end{array}$ & $\begin{array}{c}0.092^{\star \star \star}(0.025) \\
30,906\end{array}$ & $\begin{array}{c}0.067^{\star \star \star}(0.025) \\
30,519\end{array}$ \\
\hline
\end{tabular}




\begin{tabular}{|c|c|c|c|c|}
\hline & \multicolumn{2}{|c|}{ Neglect } & \multicolumn{2}{|c|}{ Physical Abuse } \\
\hline & (1) & (2) & (1) & (2) \\
\hline \multicolumn{5}{|c|}{ Mental Health Problem: Depression } \\
\hline $\begin{array}{l}\text { All ages } \\
(\text { Mean=0.09) }\end{array}$ & $\begin{array}{c}0.055^{\star \star \star}(0.009) \\
32,422\end{array}$ & $\begin{array}{c}0.036^{\star \star \star}(0.009) \\
32,004\end{array}$ & $\begin{array}{c}0.170^{* \star \star}(0.029) \\
32,110\end{array}$ & $\begin{array}{c}0.131^{\star \star \star}(0.029) \\
31,704\end{array}$ \\
\hline $\begin{array}{l}\text { By age } 30 \\
(\text { Mean=0.02) }\end{array}$ & $\begin{array}{c}0.013^{\star \star \star}(0.005) \\
31,569\end{array}$ & $\begin{array}{c}0.009 *(0.005) \\
31,174\end{array}$ & $\begin{array}{c}0.028^{\star}(0.015) \\
31,263\end{array}$ & $\begin{array}{c}0.015(0.015) \\
30,880\end{array}$ \\
\hline $\begin{array}{l}\text { By age } 40 \\
(\text { Mean }=0.03)\end{array}$ & $\begin{array}{c}0.020^{\star \star \star}(0.006) \\
31,569\end{array}$ & $\begin{array}{c}0.014^{\star \star}(0.006) \\
31,174\end{array}$ & $\begin{array}{c}0.044^{\star \star}(0.019) \\
31,263\end{array}$ & $\begin{array}{c}0.024(0.019) \\
30,880\end{array}$ \\
\hline $\begin{array}{l}\text { By age } 50 \\
(\text { Mean }=0.05)\end{array}$ & $\begin{array}{c}0.035^{\star \star \star}(0.007) \\
31,569\end{array}$ & $\begin{array}{c}0.020^{\star \star \star}(0.007) \\
31,174\end{array}$ & $\begin{array}{c}0.119^{\star \star \star}(0.027) \\
31,263\end{array}$ & $\begin{array}{c}0.088^{\star \star \star \star}(0.026) \\
30,880\end{array}$ \\
\hline $\begin{array}{l}\text { By age } 60 \\
(\text { Mean=0.06) }\end{array}$ & $\begin{array}{c}0.043^{\star \star \star}(0.008) \\
31,569\end{array}$ & $\begin{array}{c}0.028^{\star \star \star}(0.008) \\
31,174\end{array}$ & $\begin{array}{c}0.132^{* \star \star}(0.028) \\
31,263\end{array}$ & $\begin{array}{c}0.103^{\star \star \star}(0.028) \\
30,880\end{array}$ \\
\hline $\begin{array}{l}\text { By age } 70 \\
\text { (Mean=0.06) }\end{array}$ & $\begin{array}{c}0.044^{\star \star \star}(0.008) \\
31,569\end{array}$ & $\begin{array}{c}0.030^{\star \star \star}(0.008) \\
31,174\end{array}$ & $\begin{array}{c}0.136^{\star \star \star}(0.029) \\
31,263\end{array}$ & $\begin{array}{c}0.107^{\star \star \star}(0.028) \\
30,880\end{array}$ \\
\hline
\end{tabular}

Note: The table contains estimates from linear regression models with robust standard errors. Each cell contains results from a different model. Within each cell, we first report the estimated coefficients; we then report in parentheses robust standard errors; we last report in italics the sample size. For each sweep, outcome and exposure, we report two different specifications, in the columns (1) to (2), respectively. Specification (1) only includes as controls a basic set of demographics: age, gender, ethnicity and the month and year of the interview. Specification (2) adds a set of adverse early childhood experiences: self-reported health in childhood (excellent, very good or good health compared to fair or poor), whether missed school for over a month due to health as child, whether in hospital for more than a month due to health as child, whether when aged $<16$ parents ever permanently separated or divorced, whether when aged $<16$ either parents unemployed for over $6 \mathrm{mths}$ when wanted to work, whether when aged $<16$ parents argue or fight very often, whether when aged $<16$ ever separated from mother for 6 months or more, whether when aged $<16$ parents drunk/took drugs/had mental health problems. 
Table 21: ELSA Results of the Effects of CM on Healthy Behaviours, Labour Market Outcomes and Welfare Use

\begin{tabular}{|c|c|c|c|c|}
\hline & \multicolumn{2}{|c|}{ Neglect } & \multicolumn{2}{|c|}{ Physical Abuse } \\
\hline & (1) & $(2)$ & (1) & $(2)$ \\
\hline \multicolumn{5}{|c|}{ Healthy Behaviours: Heavy Drinking } \\
\hline $\begin{array}{l}\text { All Ages } \\
(M e a n=0.14)\end{array}$ & $\begin{array}{c}0.027^{\star \star \star}(0.009) \\
31,372 \\
\end{array}$ & $\begin{array}{c}0.032^{\star * \star}(0.010) \\
30,964 \\
\end{array}$ & $\begin{array}{c}-0.033^{\star \star}(0.016) \\
31,101 \\
\end{array}$ & $\begin{array}{c}-0.031 *(0.018) \\
30,705 \\
\end{array}$ \\
\hline \multicolumn{5}{|c|}{ Healthy Behaviours: Current Smoker } \\
\hline $\begin{array}{l}\text { All Ages } \\
(\text { Mean=0.13) }\end{array}$ & $\begin{array}{c}0.013(0.009) \\
33,901\end{array}$ & $\begin{array}{c}0.004(0.010) \\
33,463\end{array}$ & $\begin{array}{c}0.080^{\star \star \star}(0.025) \\
33,581\end{array}$ & $\begin{array}{c}0.063^{* \star}(0.026) \\
33,155\end{array}$ \\
\hline \multicolumn{5}{|c|}{ Healthy Behaviours: Heavy Smoker (25 Cigarettes/Day or More) } \\
\hline $\begin{array}{l}\text { All Ages } \\
(\text { Mean=0.08) }\end{array}$ & $\begin{array}{c}0.009(0.015) \\
4,325\end{array}$ & $\begin{array}{c}0.006(0.016) \\
4,250\end{array}$ & $\begin{array}{c}0.027(0.033) \\
4,260 \\
\end{array}$ & $\begin{array}{c}0.023(0.035) \\
4,191\end{array}$ \\
\hline \multicolumn{5}{|c|}{ Labour Market Outcome: Employed } \\
\hline $\begin{array}{l}\text { All Ages } \\
\text { (Mean=0.35-0.36) }\end{array}$ & $\begin{array}{c}-0.013(0.009) \\
34,003\end{array}$ & $\begin{array}{c}-0.008(0.010) \\
33,564\end{array}$ & $\begin{array}{c}-0.051^{\star \star}(0.024) \\
33,682\end{array}$ & $\begin{array}{c}-0.037(0.025) \\
33,255\end{array}$ \\
\hline \multicolumn{5}{|c|}{ Labour Market Outcome: Weekly Earnings (if Employed) } \\
\hline $\begin{array}{l}\text { All Ages } \\
\text { (Mean=278-279) }\end{array}$ & $\begin{array}{c}-8.992(9.811) \\
12,069\end{array}$ & $\begin{array}{c}-1.63(9.85) \\
11,926\end{array}$ & $\begin{array}{c}-0.30(20.42) \\
11,974\end{array}$ & $\begin{array}{c}17.85(21.30) \\
11,831\end{array}$ \\
\hline \multicolumn{5}{|c|}{ Labour Market Outcome: Weekly Earnings (AlI) } \\
\hline $\begin{array}{l}\text { All Ages } \\
\text { (Mean=100) }\end{array}$ & $\begin{array}{c}-16.12(12.16) \\
33,938\end{array}$ & $\begin{array}{c}-7.17(12.68) \\
33,499\end{array}$ & $\begin{array}{c}-52.94^{\star}(29.08) \\
33,620\end{array}$ & $\begin{array}{c}-28.25(30.23) \\
33,193\end{array}$ \\
\hline \multicolumn{5}{|c|}{ Labour Market Outcome: Weekly Disability-Related Benefits (if Participants) } \\
\hline $\begin{array}{l}\text { All Ages } \\
(\text { Mean=85) }\end{array}$ & $\begin{array}{c}11.24(9.92) \\
2,788 \\
\end{array}$ & $\begin{array}{c}13.17(13.82) \\
2,744 \\
\end{array}$ & $\begin{array}{c}41.88(31.65) \\
2,762 \\
\end{array}$ & $\begin{array}{c}50.24(42.66) \\
2,724 \\
\end{array}$ \\
\hline \multicolumn{5}{|c|}{ Labour Market Outcome: Weekly Disability-Related Benefits (AII) } \\
\hline $\begin{array}{l}\text { All Ages } \\
(\text { Mean=7) }\end{array}$ & $\begin{array}{c}40.37^{\star \star \star}(15.25) \\
33,806\end{array}$ & $\begin{array}{c}30.66^{\star \star}(15.15) \\
33,370\end{array}$ & $\begin{array}{c}131.88^{\star \star \star}(43.74) \\
33,490\end{array}$ & $\begin{array}{c}111.02^{\star \star}(43.83) \\
33,066\end{array}$ \\
\hline
\end{tabular}

Note: The table contains estimates from linear regression models with robust standard errors, apart from the outcomes "weekly earnings (all)" and "weekly disability-related benefits (all)", which are estimated using tobit models. Each cell contains results from a different model. Within each cell, we first report the estimated coefficients; we then report in parentheses robust standard errors; we last report in italics the sample size. For each sweep, outcome and exposure, we report two different specifications, in the columns (1) to (2), respectively. Specification (1) only includes as controls a basic set of demographics: age, gender, ethnicity and the month and year of the interview. Specification (2) adds a set of adverse early childhood experiences: self-reported health in childhood, whether missed school for over a month due to health as child, whether in hospital for more than a month due to health as child, whether when aged $<16$ parents ever permanently separated or divorced, whether when aged $<16$ either parents unemployed for over $6 \mathrm{mths}$ when wanted to work, whether when aged $<16$ parents argue or fight very often, whether when aged $<16$ ever separated from mother for 6 months or more, whether when aged $<16$ parents drunk/took drugs/had mental health problems. 
Table 22: ELSA Results of the Effects of the global measure of CM

(1)

(2)

\begin{tabular}{|c|c|c|}
\hline \multicolumn{3}{|c|}{ Physical Health Problem: Obesity } \\
\hline All ages (HSE) & $0.019(0.014)$ & $0.023(0.015)$ \\
\hline \multicolumn{3}{|c|}{ Physical Health Problem: Hypertension } \\
\hline All ages & $0.015(0.013)$ & $0.017(0.014)$ \\
\hline \multicolumn{3}{|c|}{ Physical Health Problem: Diabetes } \\
\hline All ages & $0.009(0.008)$ & $0.010(0.009)$ \\
\hline \multicolumn{3}{|c|}{ Physical Health Problem: Cancer } \\
\hline All ages & $0.001(0.007)$ & $0.001(0.007)$ \\
\hline \multicolumn{3}{|c|}{ Mental Health Problem: Emotional/psychotic disorder (all types) } \\
\hline All ages & $0.069 * \star \star(0.009)$ & $0.045^{\star \star \star}(0.010)$ \\
\hline By age 30 & $0.013^{\star \star}(0.005)$ & $0.008(0.005)$ \\
\hline By age 40 & $0.022^{\star \star \star}(0.006)$ & $0.014^{\star \star}(0.006)$ \\
\hline By age 50 & $0.042^{\star \star *}(0.008)$ & $0.026^{\star \star *}(0.008)$ \\
\hline By age 60 & $0.050 * \star * * 0.009)$ & $0.033^{* \star \star}(0.009)$ \\
\hline By age 70 & $0.051^{\star \star *}(0.009)$ & $0.034^{\star \star *}(0.009)$ \\
\hline \multicolumn{3}{|c|}{ Mental Health Problem: Anxiety } \\
\hline All ages & $0.056^{\star \star \star}(0.008)$ & $0.037^{\star \star \star}(0.008)$ \\
\hline By age 30 & $0.010^{\star \star}(0.004)$ & $0.007(0.005)$ \\
\hline By age 40 & $0.018^{\star \star \star}(0.005)$ & $0.012^{\star \star}(0.006)$ \\
\hline By age 50 & $0.032^{\star \star *}(0.007)$ & $0.020^{\star \star \star}(0.007)$ \\
\hline By age 60 & $0.038^{\star \star \star}(0.007)$ & $0.025^{\star \star \star}(0.008)$ \\
\hline By age 70 & $0.039^{\star \star \star}(0.007)$ & $0.026^{\star \star \star}(0.008)$ \\
\hline \multicolumn{3}{|c|}{ Mental Health Problem: Depression } \\
\hline All ages & $0.058^{\star \star *}(0.009)$ & $0.038^{* * \star}(0.009)$ \\
\hline By age 30 & $0.013^{\star \star \star}(0.005)$ & $0.009 *(0.005)$ \\
\hline By age 40 & $0.022^{\star \star *}(0.006)$ & $0.015^{\star \star}(0.006)$ \\
\hline By age 50 & $0.039 * \star \star(0.007)$ & $0.022^{* * *}(0.007)$ \\
\hline By age 60 & $0.046^{\star \star \star}(0.008)$ & $0.030^{* \star *}(0.008)$ \\
\hline By age 70 & $0.047^{\star \star \star}(0.008)$ & $0.031^{* \star \star}(0.008)$ \\
\hline
\end{tabular}

Note: The table contains estimates from linear regression models (robust standard errors in parentheses). We report results for two different specifications. Specification (1) (left column) only includes as controls a basic set of demographics: age, gender, ethnicity and the month and year of the interview. Specification (2) (right column) adds a set of adverse early childhood experiences: selfreported health in childhood, whether missed school for over a month due to health as child, whether in hospital for more than a month due to health as child, whether when aged $<16$ parents ever permanently separated or divorced, whether when aged $<16$ either parents unemployed for over $6 \mathrm{mths}$ when wanted to work, whether when aged $<16$ parents argue or fight very often, whether when aged $<16$ ever separated from mother for 6 months or more, whether when aged $<16$ parents drunk/took drugs/had mental health problems. 
Table 23: ELSA Results of the Effects of the global measure of CM

(1)

(2)

\begin{tabular}{|c|c|c|}
\hline \multicolumn{3}{|c|}{ Healthy Behaviours: Heavy Drinking } \\
\hline All ages & $0.026^{\star \star \star}(0.009)$ & $0.029^{\star \star \star}(0.009)$ \\
\hline \multicolumn{3}{|c|}{ Healthy Behaviours: Current Smoker } \\
\hline All ages & $0.016^{*}(0.009)$ & $0.006(0.010)$ \\
\hline \multicolumn{3}{|c|}{ Healthy Behaviours: Heavy Smoker (25 Cigarettes/Day or More) } \\
\hline All ages & $0.012(0.015)$ & $0.010(0.016)$ \\
\hline \multicolumn{3}{|c|}{ Labour Market Outcome: Employed } \\
\hline All ages & $-0.017^{*}(0.009)$ & $-0.010(0.010)$ \\
\hline \multicolumn{3}{|c|}{ Labour Market Outcome: Weekly Earnings (if Employed) } \\
\hline All ages & $-8.17(10.02)$ & $1.95(9.94)$ \\
\hline \multicolumn{3}{|c|}{ Labour Market Outcome: Weekly Earnings (All) } \\
\hline All ages & $-18.73(12.46)$ & $-6.98(12.94)$ \\
\hline \multicolumn{3}{|c|}{ Labour Market Outcome: Weekly Disability-Related Benefits (if Participants) } \\
\hline All ages & $12.47(9.31)$ & $14.02(13.79)$ \\
\hline \multicolumn{3}{|c|}{ Labour Market Outcome: Weekly Disability-Related Benefits (AII) } \\
\hline All ages & $47.49^{\star \star \star}(16.18)$ & $35.59^{\star \star}(15.60)$ \\
\hline
\end{tabular}

Note: The table contains estimates from linear regression models with robust standard errors, apart from the outcomes "weekly earnings (all)" and "weekly disability-related benefits (all)", which are estimated using tobit models. Each cell contains results from a different model. Within each cell, we first report the estimated coefficients; we then report in parentheses robust standard errors; we last report in italics the sample size. For each sweep, outcome and exposure, we report two different specifications, in the columns (1) to (2), respectively. Specification (1) only includes as controls a basic set of demographics: age, gender, ethnicity and the month and year of the interview. Specification (2) adds a set of adverse early childhood experiences: self-reported health in childhood, whether missed school for over a month due to health as child, whether in hospital for more than a month due to health as child, whether when aged $<16$ parents ever permanently separated or divorced, whether when aged $<16$ either parents unemployed for over $6 \mathrm{mths}$ when wanted to work, whether when aged $<16$ parents argue or fight very often, whether when aged $<16$ ever separated from mother for 6 months or more, whether when aged $<16$ parents drunk/took drugs/had mental health problems. 


\section{The long-term economic consequences of child maltreatment in the UK}

\subsection{Aims}

The aim of this part of the study is to calculate new estimates of the lifetime costs per victim of nonfatal and fatal child maltreatment from a societal perspective in the UK using an incidence-based approach. We have tried to quantify these costs as far as is possible, and have focused on the financial costs of child maltreatment in terms of its impact on health care, social care, education, the criminal justice system, and the wider economy in terms of lost productivity. We have not been able to include intangible costs such as the emotional pain borne by victims, though we recognise these are substantial. We use the best available evidence to develop our estimates, and the analysis is informed by the econometric analysis described previously. Given limitations of the data, there is uncertainty in our estimates. We have been conservative in the assumptions we have made, and undertook extensive sensitivity analyses to test the impact of the uncertainty on our findings.

\subsection{Methods}

\section{Costs included in the analysis}

We assessed the lifetime cost of child maltreatment in the UK adopting a societal perspective, including costs to the health care, social care, education and criminal justice sectors, and to the wider economy in terms of lost productivity. Based on the literature review and analyses described above we included: short-term health-related costs; long-term healthrelated costs; criminal justice costs; childrens' social care system costs; special education costs; and, productivity losses due to reduced employment.

Short-term health-related costs include costs associated with unplanned hospital admissions for injuries, and health care and criminal justice costs associated with mental health disorders arising from child maltreatment.
Long-term health related costs include costs due to depression, anxiety, smoking and alcohol abuse, which were found to be related to child maltreatment (the global measure) in the econometric analysis. We did not include costs associated with obesity, hypertension, type 2 diabetes, raised cholesterol, cancer and height as these were not significantly related to the global measure of child maltreatment. We have included costs of other conditions that are related to depression, anxiety, smoking and alcohol abuse where these are included in published estimates (e.g., the cost of developing lung cancer among smokers is included in the smoking-related costs).

Productivity losses might arise from child maltreatment due to premature mortality, days off work, reduced employment and reduced wages among those who are employed. In our analysis productivity losses were captured in two ways. First, in terms of reduced employment. This was based on the findings of the econometric analysis. Second, we included productivity losses arising from days off work and premature mortality due to long-term health-related problems associated with child maltreatment. We were not able to evaluate days off work among those who are employed arising from child maltreatment directly, as these data were not available in the datasets we used for our analysis, but we did include these costs in our calculation of long-term health-related costs where they were included in published cost estimates (e.g., absenteeism due to depression). To avoid double counting, when we included productivity losses from long-term health-related problems we removed the productivity losses due to reduced unemployment. We found that child maltreatment was not associated with lower wages among those who were employed, so we did not include this effect.

We did not include costs associated with drug use as we did not have data on this in our econometric analysis. We did not evaluate the impact of child 
maltreatment on education attainment, but we included special education costs associated with child maltreatment and accounted for education effects indirectly in our calculation of productivity losses. We did not evaluate the impact of child maltreatment on the likelihood and cost of divorce as it was unclear how we would value these costs. Our econometric analysis showed that child maltreatment is associated with increased disability throughout the life course and the receipt of disability benefits. Health-related costs are included in our analysis but we did not include disability benefits as these are transfer payments ${ }^{20}$ (Hodgson and Meiners 1982, Luce, Manning et al. 1996, Choi, Robson et al. 1997).

\section{Costing methodology}

We used the econometric analysis described above, supplemented with the limited but best available published evidence to develop our cost estimates. We used the findings from the analysis of the global measure of child maltreatment, and preferred the NCDS results over the ELSA results as the former included more types of child maltreatment. The only exception to this is that we used the ELSA results for heavy drinking as this was felt to be a more appropriate measure of alcohol abuse than the NCDS measure. We used the coefficients from the most detailed econometric model (specification (4) of the NCDS analysis; (2) of the ELSA analysis). While some relevant variables were available in the datasets we analysed to measure the short-terms costs described above the data were not sufficiently detailed (e.g., in terms of the timing of child maltreatment). Therefore we based our calculations of short-term costs on published figures. Secondary evidence was identified via searches of several databases including PubMed, the NHS Economic Evaluations Database, EconLit, Google Scholar and Google. For each cost category we used published estimates from previous cost of illness studies where possible. We used data that were specific to the UK as opposed to countries outside of the UK because health care, social care, education and criminal justice systems vary between countries and costs in other countries are unlikely to apply to the UK. The only exception to this was that in sensitivity analyses we applied costs of child maltreatment calculated for the USA by Fang and colleagues (Fang, Brown et al. 2012) to the UK to compare differences between the two studies. Costs vary over time and we used recent data whenever possible, though as noted below several of the data sources are dated.

We did not evaluate costs by type and severity of child maltreatment and instead opted for an overall maltreatment estimate. One reason is that many of the studies used to provide inputs into the calculations did not distinguish by type of maltreatment, so disaggregating costs would be difficult. In our econometric analysis we were able to analyse the impact of different types of maltreatment on long-term health outcomes and labour market outcomes, but small numbers of cases for some types of maltreatment meant the analysis was underpowered. Another issue is that there may be overlap between different types of maltreatment making it difficult to attribute costs to individual types of maltreatment.

For several of the cost components we required longitudinal data on the number of events for new cases of child maltreatment in a single year to be able to accurately calculate incidence-based costs. These data were not available so as noted below we were required to make 'steady-state' assumptions, as have been used by other researchers. Using this approach the number of events in a single year across all victims of child maltreatment is a proxy for all events over time among new victims in that year. This assumption requires that the number of events remains fairly constant over time, which may be problematic for some of the cost components considered here.

Our aim was to estimate the lifetime cost per victim of child maltreatment, for several of the cost components data were only available at the aggregate level across all victims. Therefore to calculate costs per victim we were required to divide

20 A transfer payment (or government transfer) is a redistribution of income. These payments do not directly absorb resources or create output, and are made without any exchange of goods or services. Disability benefits are transfer payment as they represent a payment made or an income received in which no goods or services are being paid for. The primary reason for excluding transfer payments is to avoid "double counting" as these payments are made from the government and received from beneficiaries and therefore there is no loss from a societal perspective. 
these costs through by an estimate of the number of new cases of child maltreatment. This number is uncertain.

All costs were estimated in UK£ and adjusted to the 2015 prices (our reference year) where necessary using gross domestic product deflators. ${ }^{21}$

We assumed that when children are maltreated the average age at which it starts is 6 years (Department for Education (DfE) 2016). ${ }^{22}$ We used this as the starting point to calculate lifetime costs per victim, but acknowledge there is some uncertainty with this figure. There is no data source that tells at what age abuse typically starts. Our assumption that it starts on average at 6 years of age is based on published child protection statistics that are available in each of the four nations of the UK and reflects the age children become subject to a child protection plan or come on to the child protection register. We have looked at data for England, Wales Northern Ireland and Scotland and taken weighted averages. Where age ranges were available mid-points were used to calculate weighted averages. This assumption is conservative because we know the age of the child at the end of the year as opposed to when the child become subject to a protection plan. Also the abuse will have started before children become subject to a protection plan. The abuse could have just started, or it could have been happening for a number of years.

Costs are presented in present value terms; future costs up to 30 years from the age of 6 are discounted using an annual rate of 3.5\% (National Institute for Health and Care Excellence 2013);23 for costs incurred between 31 and 74 years from age 6 we applied an annual discount rate of 3\%; for those over 75 years we applied an annual rate of $2.5 \% .^{24}$ As noted above, and described in more detail below, there is uncertainty in our estimates, reflecting data limitations. We therefore provide central estimates and undertook extensive sensitivity analysis to take this into account.

\section{Lifetime costs per victim of non-fatal child maltreatment}

\section{Short-term health-related costs}

Short-term health-related costs of child maltreatment refer to health care and associated costs up to the age of 18 . We include costs incurred by treating injuries from maltreatment and to treat mental health problems associated with maltreatment (Summers and Molyneux 1992, Hennes 2001, Corso and Fertig 2010, Brown, Fang et al. 2011, Fang, Brown et al. 2012, Florence, Brown et al. 2013, Peterson, Xu et al. 2014, Saied-Tessier 2014, Boschung 2015).

\section{Unplanned hospital admissions for injuries}

We valued short-term health care costs in terms of unplanned hospital admissions for maltreatment or violence-related injuries. According to GonzalesIzquierdo et al (2014)(Gonzalez-Izquierdo, CortinaBorja et al. 2014) between 1 January 2005 and 31 March 2012 there were 61,574 unplanned hospital admissions (ordinary admissions and day cases) for maltreatment or violence-related (MVR) injury in children aged 18 years or younger in England and Scotland, where MVR diagnoses were defined using previously published and validated methods.

We applied the unplanned admission rate for maltreatment or violence-related injuries for England in 2011 by age groups reported by Gonzales-Izquierdo et al to mid-year UK population estimates and estimated there were 8,685 unplanned admissions in 2015 in the UK. Ideally we would have longitudinal data on the number admissions for new cases of child maltreatment in a single year. These data are not available so we use a 'steady-state' methodology also used by other researchers to estimate the lifetime costs of a disease or problem when longitudinal data are not available (Barnett, Birnbaum et al. 2000, Birnbaum, Leong et al. 2003, Fang, Brown et al. 2012). Making this assumption, the total admissions in one year for all victims is a proxy for all admissions over time for

21 https://www.gov.uk/government/uploads/system/uploads/attachment_data/file/490866/GDP_Deflators_Qtrly_National_ Accounts_December_2015_update.csv/preview

22 Department for Education (DfE) 2016

23 https://www.nice.org.uk/article/pmg9/chapter/5-The-reference-case\#discounting

24 https://www.gov.uk/government/uploads/system/uploads/attachment_data/file/220541/green_book_complete.pdf 
new victims in that year. We therefore assume in the absence of data that these admissions relate to new cases of child maltreatment. The national average unit cost of non-elective long stay and non-elective short stay admissions for paediatric injuries was $£ 853$ ( $D O H$ 2015). Hence the cost of unplanned hospital admissions for maltreatment or violencerelated injuries among new cases of maltreatment in 2015 is estimated to be $8,685^{\star} £ 853=$ $£ 7,405,733$. This figure was divided by an estimate of the incidence of child maltreatment based on the number of children coming onto child protection plans or registers in 2015 minus the number of re-registrations (61,894 (Department for Education (DfE) 2016)) to give an estimate of the average cost per victim of $£ 120$.

Note this calculation assumes that admissions rates for maltreatment or violence-related injuries across the whole of the UK are the same as for England, which may not be true. Also, this figure is an underestimate of the costs of treating childmaltreatment-related injuries as it only includes hospital costs; we did not include other health care costs, e.g., from primary care contacts and outpatient attendances, as we were unable to attribute these to child maltreatment. We explore the impact of including these costs in sensitivity analyses.

\section{Short-term mental health problems}

Maltreatment is commonly associated with mental health-related responses to stress and trauma, including depression, anxiety, post-traumatic stress disorder and behavioural disorders, but UK evidence is sparse on the incremental effect of child maltreatment on mental health problems in children and young people.

To assess the incremental effect of child maltreatment on mental health problems we used the prevalence of mental health problems in children in care minus the prevalence of those not in care (Meltzer, Gatward et al. 2003). This assumes the prevalence of mental health problems for children who have been maltreated is the same as those in care. In a study of mental health problems, service use and costs among children in foster care, $93 \%$ of the sample had suffered some form of abuse or neglect (Minnis, Everett et al. 2006). This approach also assumes that if those who were maltreated were not maltreated then the prevalence in this group would be the same as those not in care. Using this approach, the incremental probability of suffering emotional disorders, conduct disorders and hyperkinetic disorders is summarised in Table 24 by age group. In the absence of data we assumed the effect in children aged $11-15$ years applies to those up to the age of 18 .

Table 24. Marginal effect of child maltreatment on probability of mental health problems during childhood

\begin{tabular}{lcc}
\hline Disorder & $\begin{array}{c}\text { Children age } \\
\text { 5-10 years }\end{array}$ & $\begin{array}{c}\text { Children age 11- } \\
\text { 15 years }\end{array}$ \\
\hline Emotional disorder & 0.08 & 0.059 \\
\hline Conduct disorder & 0.315 & 0.345 \\
\hline Hyperkinetic disorder & 0.091 & 0.061 \\
\hline
\end{tabular}

We combined these figures with costs reported by Snell and colleagues (Snell, Knapp et al. 2013). Mean annual costs of hyperkinetic disorders, conduct disorders and emotional disorders in 2015 prices were $£ 3,605, £ 2,153$ and $£ 1,351$, respectively, including primary care, paediatric health services and mental services costs.

We then estimated the incremental cost of child maltreatment on mental health problems in children and young people by multiplying the estimated incremental effect of child maltreatment on the probability of each disorder by the annual costs for each year from 6 to 18 years. Summing across the different types of disorder the total discounted costs were $£ 11,453$ per victim up to age 18 (i.e., over 12 years).

There is evidence that maltreatment may lead to increased delinquency and crime (Rutter 1998), though data for the UK are limited. We estimated criminal justice system costs attributable to mental health problems in children and young people based on mean criminal justice system costs per year associated with conduct disorder reported by Scott and colleagues (Scott, Knapp et al. 2001), (£3,436 in 2015 prices). We multiplied this figure by the increased probability of conduct disorder 
associated with child maltreatment in Table 24. Applying these figures from ages 6 to 18, the discounted cost was $£ 7,100$ per victim (i.e., over 12 years).

The total cost of mental health problems in maltreated children is the sum of the health service and criminal justice system costs, or $£ 18,553$ per victim. The main uncertainty in this analysis is the assumption concerning the impact of child maltreatment on mental health problems. We explore this further in sensitivity analysis. There is also evidence that mental health problems in childhood are associated with significant societal costs in adulthood, but we included some of these costs in the long-term health-related costs below.

\section{Long term health-related costs}

We considered the long-term impact of child maltreatment on anxiety, depression, smoking and alcohol abuse in adulthood based on the findings of the econometric analysis described in the previous section.

\section{Anxiety}

McCrone and colleagues (McCrone 2008)

calculated that the average cost of anxiety per year per person with anxiety who is in contact with the health service is $£ 1,282$ (2015 prices). This includes direct health and social care costs, informal care costs and criminal justice system costs. McCrone and colleagues also report productivity losses due to anxiety, but these are attributed to the impact of anxiety on the probability of employment and so we do not include them to avoid double counting. Fineberg and colleagues (Fineberg, Haddad et al. 2013)calculated the total societal cost of anxiety per patient and reported that $46.4 \%$ of the total was due to productivity losses arising from work absence and early retirement; from this study we calculated that the productivity losses due to anxiety are $£ 661$ per person (2015 prices).

The results of the NCDS analysis using the global CM measure show that child maltreatment was positively correlated with anxiety at ages 33, 42, 50 and 55. There was a non-significant effect at age 23 (appendix, Table A2); we therefore assumed the marginal effect of child maltreatment on the probability of anxiety was zero at this age. The marginal effect of child maltreatment at each year of age over the lifetime was calculated by assuming a straight line relation between the marginal effects at ages 23, 33, 42, 50 and 55. We assumed the effect of child maltreatment before the age of 23 was captured by the short-term health-related costs described above and so did not include an impact here. We assumed the marginal effect at age 55 was constant over the remaining lifetime.

The mean incremental lifetime cost of anxiety in maltreated children was calculated as the product of the average costs per year (health care costs and productivity losses) and the marginal effect of child maltreatment on the probability of anxiety at each year of age. Due to retirement, after age 67 we did not include productivity losses.

Using this approach, the average discounted lifetime incremental cost of anxiety per victim of child maltreatment was estimated to be $£ 954$.

\section{Depression}

To estimate the costs of depression associated with child maltreatment we used the same approach as for anxiety, described above. McCrone and colleagues (McCrone 2008) calculated that the cost of depression per year per person with depression who is in contact with the health service was $£ 2,418$ (2015 prices). The cost of depression includes direct health and social care costs, informal care costs and criminal justice system costs. Productivity losses due to depression were taken from Thomas and colleagues (Thomas 2000)who calculated that costs of lost productivity with depression due to days off work and premature mortality were $£ 4,479$ per person with depression per year (2015 prices).

The NCDS analysis showed that child maltreatment was positively correlated with depression at ages 33, 42, 50 and 55, but not at age 23 (appendix, Table A2), and we applied the same methodology to calculate the marginal effect of child maltreatment on depression at each year of age.

The discounted lifetime incremental cost of depression per victim of child maltreatment was calculated to be $£ 5,145$. 


\section{Smoking}

Action on Smoking and Health (ASH)(Reckoner $2016)^{25}$ recently estimated annual costs to society due to smoking. These included the cost to the NHS and social care services of smoking-related health problems, plus costs to society due to smokingrelated fires, passive smoking and productivity losses due to smoking breaks, days off sick and early deaths. The total cost in England in 2015 was estimated to be $£ 13.9$ billion. The authors also reported an estimated smoking population in England of 7,687,769, indicating a mean societal cost per smoker per year of $£ 1,805$ (2015 prices).

Our NCDS analysis showed that child maltreatment was positively correlated with being a current smoker at ages 33, 42 and 50 but not at ages 23 (appendix, Table A2) and 55. We assumed the marginal effect of child maltreatment on smoking was zero at ages 23 and 55. The effect of child maltreatment over the lifetime was calculated by assuming a straight line relation between marginal effects at ages 23, 33, 42, 50 and 55. We assumed the effect of child maltreatment on smoking before the age of 23 was zero and that the marginal effect of zero at age 55 was constant for the remaining lifetime.

The mean incremental lifetime cost of smoking in maltreated children was calculated by multiplying the cost per smoker per year by the marginal effect of child maltreatment at each year of age. These were then discounted and summed across the lifetime, yielding a discounted lifetime incremental cost of smoking per victim of child maltreatment of $£ 528$.

\section{Alcohol abuse \\ The Cabinet Office (2003) calculated the cost of alcohol misuse to society in England at $£ 11.9$ billion per annum (2001 prices), and reported there were $9,114,371$ alcohol misusers in the same time period. The costs included NHS costs, lost productivity and crime-related costs. The lost productivity costs included the impact of reduced employment. We removed this element from the total to avoid double counting. We then inflated the figures to 2015 prices and calculated the average cost per alcohol misuser per year to be $£ 2,563$.}

According to our ELSA analysis, the impact of child maltreatment on the probability of heavy drinking (consuming 2 or more alcoholic drinks a day) was positive and statistically significant. The dataset comprised survey participants aged 50 years or more and we therefore assumed the effect of child maltreatment on alcohol misuse starts at age 50 and remains constant over the remaining lifetime. We assumed the productivity losses apply only up to an assumed retirement age of $67 .{ }^{26}$

We assumed the measure of heavy drinking in the ELSA data was commensurate with the definition of alcohol misuse used to generate the cost estimates and calculated the lifetime cost as the product of the cost per year of alcohol misuse and the marginal effect of child maltreatment on heavy drinking. Future costs were discounted and summed across the lifetime. On this basis the average discounted lifetime incremental cost of alcohol abuse per victim of child maltreatment was estimated to be $£ 537$. This figure is probably an underestimate as it assumes there is no impact of child maltreatment on problem drinking before 50 years of age.

\section{Criminal justice system costs}

Criminal justice system costs included in this category relate to the costs for police, court and penal services for the perpetrators of child abuse. As indicated, criminal justice system costs associated with the consequences of child maltreatment among victims is included in other cost categories. We based our estimates on sanction figures for child sexual abuse, because criminal justice data for other forms of maltreatment were not available. Not all cases of abuse will result in formal criminal proceedings or convictions, therefore proportions were calculated as the ratio of the number of proceeding and convictions to the total number of offences.

In 2013 police recorded 23,300 cases of sexual offences against children in the UK (Saied-Tessier 2014). In 2013 (the most recent year data are available) there were 4,100 court proceedings and 2,500 convictions registered for sexual offences against children (Saied-Tessier 2014). In the previous year 1,034 people sentenced for child 
sexual abuse were admitted onto sexual offender programmes. We calculated the proportion of sexual offence cases that resulted in court proceedings and convictions and applied these proportions to the total number of criminal cases for sexual offences, abuse and neglect against children in 2015. We also applied the proportion of sexual offence cases that led to admission onto sexual offenders programmes in 2013 to the number of criminal cases of sexual offences against children in 2015. The resulting figures, shown in Table 25, were multiplied by the unit costs of court proceedings, convictions and sex offender treatment programmes (shown in Table 25 in 2015 prices) and summed to calculate total criminal justice system costs. Note the proportion of offences resulting in referral to sex offenders programmes is applied only to sexual abuse cases.

Note that as only a limited number of individuals can be accommodated on sexual offenders programmes the costs reported here are likely to underestimate the financial input needed.

Similar to our treatment of the injury costs above, we make a steady-state assumption and assume the new offences apply to new cases of child maltreatment. Using this assumption, the total number of new offences in one year is used as a proxy for the lifetime number of offences relating to new cases of child maltreatment in that years. This approach also assumes, in the absence of data, that the proportion of prosecuted cases for all type of maltreatment are the same as for sexual offences.

This analysis includes criminal justice costs arising from non-fatal child maltreatment cases. Costs associated with fatal child maltreatment are considered separately below.

Using this approach, the criminal justice system costs due to new cases of child maltreatment in 2015 are estimated to be $£ 267,148,144$. This figure was divided by an estimate of the incidence of child maltreatment in 2015 (61,894 (Department for Education (DfE) 2016)) to give an estimate of the average cost per victim of $£ 4,316$.
Table 25. Criminal justice system costs due to new cases of child maltreatment in 2015

\begin{tabular}{lccc}
\hline & Number & $\begin{array}{c}\text { Unit cost } \\
(£)\end{array}$ & $\begin{array}{c}\text { Total cost } \\
(£)\end{array}$ \\
\hline Court proceedings & 10030 & 11,746 & $117,819,570$ \\
\hline Convictions & 6116 & 20,362 & $124,535,565$ \\
\hline $\begin{array}{l}\text { Sex offenders } \\
\text { treatment } \\
\text { programmes }\end{array}$ & 2249 & 11,025 & $24,7923,010$ \\
\hline $\begin{array}{l}\text { Total criminal } \\
\text { justice cost }\end{array}$ & & & \\
\hline
\end{tabular}

\section{Child social care costs}

Child social care costs include the cost of being on a child protection plan or register (initial referral and assessment once maltreatment has been identified, plus ongoing support), and the costs of foster care and local authority residential home care among those who receive it. According to recent data $61 \%$ of looked after children in England and $66 \%$ in Wales are looked after due to maltreatment. (NSPCC, 2016).

We multiplied the number of children coming onto child protection plans or registers in 2015 minus the number of re-registrations (61,894 (Department for Education (DfE) 2016)) by the fixed costs and ongoing support costs obtained from published sources (inflated to 2015 prices; Table 26). For the ongoing support costs we assumed that all children would receive support for at least 6 months and a proportion would receive support for up to two years. The total amount of the fixed cost is $£ 329,345,366$, and the total ongoing costs in the UK for the first 6 months are estimated to be $£ 121,752,925$, with an additional $£ 13,684,204$ for those children staying on a plan for at least 2 years.

We assume that $78 \%$ of newly looked after children are put into foster care and $14 \%$ are placed in a local authority care home (including residential care homes, secure units, homes and hostels, and residential schools), based on English data for all newly-looked after children, irrespective of the cause in 2014 (Department for Education (DfE) 2015). ${ }^{27}$ We applied this to figures on the total number of newly looked after children in the UK

27 https://www.gov.uk/government/uploads/system/uploads/attachment_data/file/464756/SFR34_2015_Text.pdf https://www.gov.uk/government/statistics/children-looked-after-in-england-including-adoption-2014-to-2015 
that year. We assume that each child spends an average period of 785 days in foster care or local authority care, based on data from the same source. The costs of being placed for adoption, placement with parents, other placements in the community, and other placements are not known and therefore not included. . We calculated the discounted cost per child receiving foster care, local authority care and multiplied these by the estimated numbers of newly looked after maltreated children receiving each intervention. The total discounted costs were $£ 982,460,996$ and $£ 912,886,189$ respectively (Table 26).

The total cost of child social care is estimated to be $£ 2,360,129,680$. This figure was divided by the estimate of the incidence of child maltreatment in 2015 (61,894 (Department for Education (DfE) 2016)) to give an estimate of the average cost per victim of $£ 38,132$.

\section{Special education costs}

There is evidence that maltreated children are more likely to receive special education support (Fang, Brown et al. 2012, Fisher 2016). Fisher (2016) calculated that the proportion of children using special education services who were reported to have been severely maltreated (physical abuse, sexual abuse, emotional abuse or neglect or physical neglect) up to 12 years of age was $42 \%$, compared to $20 \%$ among children who were not reported to be maltreated. Therefore, the incremental effect of child maltreatment on receipt of special education was 22 percentage points (unadjusted).

The Department for Children, Schools and Families reports that $£ 3,310$ per pupil was spent on the provision of education for children with special educational needs in England in 2008-2009 (DCSF January Schools Census 2009). Inflating this value to 2015 we assume every child incurred costs of $£ 3,740$ in 2015 for special education services.

Table 26. Child social care costs due to maltreatment in 2015

\begin{tabular}{|c|c|c|c|c|c|c|}
\hline Child social care & $\begin{array}{l}\text { Percentage newly } \\
\text { looked after } \\
\text { children receiving } \\
\text { intervention (\%) }\end{array}$ & Number & Unit cost $(£)$ & $\begin{array}{l}\text { Duration } \\
\text { (days) }\end{array}$ & $\begin{array}{l}\text { Cost per child } \\
(£, \text { discounted })\end{array}$ & $\begin{array}{l}\text { Total cost } \\
(£, \text { discounted })\end{array}$ \\
\hline Foster care & 78 & 14,518 & $\begin{array}{l}88 \\
\text { (per day) }\end{array}$ & 785 & 67,672 & $982,460,996$ \\
\hline Local authority care & 14 & 2,609 & $\begin{array}{l}455 \\
\text { (per day) }\end{array}$ & 785 & 349,895 & $912,886,189$ \\
\hline \multicolumn{7}{|l|}{$\begin{array}{l}\text { Child Protection } \\
\text { Plan }\end{array}$} \\
\hline Fixed cost ${ }^{a}$ & 100 & 61,894 & $\begin{array}{l}5,321 \\
\text { (per child) }\end{array}$ & & 5,321 & $329,345,366$ \\
\hline $\begin{array}{l}\text { Ongoing support } \\
\text { costs ( } 6 \text { months) }\end{array}$ & 100 & 61,894 & $\begin{array}{l}328 \\
\text { (per month) }\end{array}$ & First 6 months & 1,967 & $121,752,925$ \\
\hline $\begin{array}{l}\text { Ongoing support } \\
\text { costs after first } 6 \\
\text { months }\end{array}$ & $\begin{array}{l}\text { 3.7\% England, Wales; } \\
\text { 3.4\% Scotland; } \\
\text { 8.6\% Northern } \\
\text { Ireland }\end{array}$ & 2,372 & $\begin{array}{l}328 \\
\text { (per month) }\end{array}$ & $\begin{array}{l}\text { From month } 7 \\
\text { to month } 24\end{array}$ & 5,768 & $13,684,204$ \\
\hline
\end{tabular}

a Initial contact and referral, assessment, cost to close case, core assessment, planning and review section 47 and public law outline 
We assume that provision of special education services starts at the age maltreatment starts and these costs are incurred until students are able to leave full-time education (assumed to be age 16 years). Hence, the duration of special education provision is assumed to be 10 years. The undiscounted cost per maltreated child in receipt of special education support is $£ 32,195$, and the cost per victim is estimated to be 0.22 * $£ 32,195=£ 7,068$.

\section{Productivity losses due to reduced employment}

As discussed, in this category we include the costs of lost productivity due to reduced employment as a result of child maltreatment. Our NCDS analysis using the global CM measure showed that child maltreatment was negatively correlated with being in employment at ages 42 and 50 years, but the effects at 33 and 55 years were non-significant. This was also the case at age 23 (appendix, Table A2). We therefore assumed the marginal effect of child maltreatment on employment was zero at all ages up to 33 years and also from age 55 onwards. The effect of child maltreatment on the probability of being employed over the lifetime was calculated by assuming a straight line relation between marginal effects at ages 33, 42, 50 and 55 years.

Lost productivity due to reduced employment was valued using age-specific earnings before deduction of taxes in 2013-14 (the most recent year available) (ONS 2014), ${ }^{28}$ inflated to 2015 prices. Based on previous studies (McCrone 2008) we assumed that earnings would increase by $2 \%$ per year. The NCDS analysis showed that child maltreatment did not have a significant effect on earnings so we did not account for this in our analysis.

We calculated the value of lifetime productivity losses due to reduced employment as the product of earnings at each age and the marginal effect of child maltreatment on employment. Future costs were discounted and summed across the lifetime. On this basis the average discounted lifetime productivity loss due per victim of child maltreatment was estimated to be $£ 14,037$.

\section{Total costs and sensitivity analyses}

We calculated our central estimate of the mean total lifetime costs of non-fatal child maltreatment per victim by summing the costs per victim across the various cost components described above. We also computed the proportion of the total accounted for by each component.

As indicated, there is uncertainty in our analysis; we therefore undertook several sensitivity analyses. First, we undertook a probabilistic sensitivity analysis (PSA) and used this to calculate 95\% uncertainty intervals around the central estimate and the probability that the mean total lifetime costs of non-fatal child maltreatment per victim would be higher than a range of pre-specified values. The parameters we varied in the analysis were those we were able to assign probability distributions to, to reflect the uncertainty with each parameter value (Briggs 2006) (Table 27). We used beta distributions to model uncertainty in the probabilities, and gamma and triangular distributions to model uncertainty in costs (gamma distributions were used where standard errors around mean values were known, triangular distributions were used where they were not; where minima and maxima were not known to parameterise triangular distributions we assumed values $\pm 50 \%$ of the central estimate). A random value from the corresponding distribution for each parameter was selected. For each simulation we generated an estimate of the total lifetime costs of non-fatal child maltreatment per victim and each individual cost component. This was repeated 1000 times and the results for each simulation were noted. 95\% uncertainty intervals were calculated as the 2.5th and 97.5th percentiles of the simulated values. The distribution of values was summarised graphically and the probability the total cost was greater a range of pre-specified values was calculated as the proportion of the simulations greater than values $£ 0$ to $£ 200,000$.

We also undertook univariate deterministic sensitivity analysis varying key values within plausible ranges one at a time. This was undertaken to highlight the sensitivity of the central estimates to individual parameter values. We varied the discount rate between $0 \%$ and $5 \%$ per annum. 
For unplanned injury-related admissions we assumed one unplanned admission per victim and that $31.2 \%$ would have a readmission (Wiljaars et al, 2016), and that each admission would be associated with it 11.5 outpatient visits based on the ratio of outpatient visits to inpatient admissions in the NHS, ${ }^{29}$ and an equivalent number of GP visits.

For short-term mental health problems we varied the annual health care costs and criminal justice system costs per victim, and the impact of child maltreatment on these problems; we also examined the effect of using the short-term health-related cost estimates from Fang et al (2012) in the USA instead of our estimates. For long-term healthrelated costs we explored the impact of increasing the marginal effect of child maltreatment on each outcome, of extrapolating the effects to older and younger ages, and of using the estimates from the USA from Fang et al. (2012). For the criminal justice system costs we increased the number of cases ending in court proceedings and convictions, and used the estimates from the USA by Fang et al. (2012). For social care costs we increased the unit costs of child protection plans and monthly ongoing support, and used the estimates for child welfare costs from the USA by Fang et al. (2012). For special education costs we varied the unit cost of support and the marginal effect of child maltreatment. For reduced employment we varied the wages that would be earned to below the national average level, we varied the annual increase in wages, we explored the impact of increasing the (negative) marginal effect of child maltreatment on employment, of extrapolating the effects to older and younger ages, and of using the estimates from the USA from Fang et al. (2012). We judged that changes $£ 10,000$ higher or lower than the central estimate were substantive.

\section{Lifetime costs per death from child maltreatment}

Following Fang et al (Fang, Brown et al. 2012) we included two components to calculate the lifetime cost per victim of fatal child maltreatment: health care costs associated with fatal injuries; and, lifetime costs of lost productivity. We also included criminal justice system costs for perpetrators of fatal child maltreatment in sensitivity analyses.
We have not been able to include costs associated with protecting other children in the family or in regular contact with perpetrators.

To calculate health care costs we used data from two published studies that calculated the cost of fatal blunt trauma and penetrating trauma injuries in the UK; mean costs per fatality for these injuries were $£ 10,966$ (Christensen, Ridley et al. 2008) and $£ 4,278$, (Christensen, Nielsen et al. 2008 respectively, in $2004 / 5$ prices. These figures included the costs of transport to the hospital, hospital stays in the Accident and Emergency department, critical care unit and on the general ward, and the costs of procedures while in hospital. Updated to 2014/15 prices the values were $£ 13,863$ and $£ 5,408$, respectively. We used the lower of these two values in our central estimate and included the higher value in sensitivity analysis.

To calculate lost productivity costs we used the human capital approach and multiplied figures for mean annual earnings by age (ONS 2014) with employment rates by age (from age 16 to 67; (Official Statistics 2016)). The earnings figures were discounted to present value terms and inflated assuming a constant annual increase in earnings of $2 \%$ (McCrone 2008). The employment-adjusted earnings figures were summed across the lifetime to provide an estimate of total lifetime earnings accounting for the likelihood of employment. We assume this figure represents mean lifetime productivity and also the lost productivity that would be incurred with a fatality from maltreatment in childhood. This assumes lifetime earnings and employment for someone who died as a result of being maltreated as a child would equal national average figures if they had not died.

We did not include a probabilistic sensitivity analysis for the lifetime costs per death from child maltreatment (including 95\% confidence intervals) as it was not possible to assign probability distributions to the key parameters. We undertook univariate deterministic sensitivity analysis we varied the discount rate, health care costs, and assumptions concerning the lost productivity. We also included criminal justice costs assuming all cases would result in court proceedings and a conviction. 
Table 27. Variables, distributions and parameters included in probabilistic sensitivity analysis

\begin{tabular}{|c|c|c|c|c|c|c|c|}
\hline & $\begin{array}{l}\text { Central } \\
\text { value }\end{array}$ & SE & Distribution & $\begin{array}{l}\text { Lower } \\
\text { limit }\end{array}$ & $\begin{array}{l}\text { Upper } \\
\text { limit }\end{array}$ & $\alpha$ & $\beta$ \\
\hline \multicolumn{8}{|l|}{ Short-term health-related costs } \\
\hline \multicolumn{8}{|l|}{ Unplanned hospital admissions for injuries } \\
\hline MVR rate $<1$ year & 102.8 & 3.878 & Gamma & & & 702.84 & 0.15 \\
\hline MVR rate $1-10$ years & 23.3 & 0.612 & Gamma & & & 1448.26 & 0.02 \\
\hline MVR rate $11-18$ years & 103.3 & 1.378 & Gamma & & & 5623.02 & 0.02 \\
\hline Unit cost per admission & $£ 853$ & & Triangular & 560 & 1014 & & \\
\hline \multicolumn{8}{|l|}{ Short-term mental health problems } \\
\hline \multicolumn{8}{|l|}{ Mean annual cost of disorders in children } \\
\hline Hyperkinetic disorder & 3,605 & 783 & Gamma & & & 21.15 & 170.48 \\
\hline Conduct disorder & 2,153 & 526 & Gamma & & & 16.75 & 128.52 \\
\hline Emotional disorder & 1,352 & 177 & Gamma & & & 58.21 & 23.22 \\
\hline $\begin{array}{l}\text { Annual criminal justice cost for children with } \\
\text { conduct disorder }\end{array}$ & 3,436 & & Triangular & 1718 & 5153 & & \\
\hline
\end{tabular}

\begin{tabular}{|c|c|c|c|c|c|c|}
\hline \multicolumn{7}{|c|}{$\begin{array}{l}\text { Marginal effect of child maltreatment on } \\
\text { probability of mental health disorders in } \\
\text { children aged 5-10 }\end{array}$} \\
\hline Emotional disorder & 0.08 & Beta & 0 & 1 & 38.28 & 309.72 \\
\hline Conduct disorder & 0.315 & Beta & 0 & 1 & 127.02 & 220.98 \\
\hline Hyperkinetic disorder & 0.091 & Beta & 0 & 1 & 38.628 & 309.372 \\
\hline
\end{tabular}

\section{Marginal effect of child maltreatment on probability of mental health disorders in children aged 11-15}

\begin{tabular}{lllllll}
\hline Emotional disorder & 0.059 & Beta & 0 & 1 & 57.12 & 422.88 \\
\hline Conduct disorder & 0.345 & Beta & 0 & 1 & 194.4 & 285.6 \\
\hline Hyperkinetic disorder & 0.061 & Beta & 0 & 1 & 34.08 & 445.92 \\
\hline
\end{tabular}

\section{Criminal justice costs incurred by}

perpetrators

\begin{tabular}{|c|c|c|c|c|c|c|}
\hline Unit cost of court proceedings & 11,746 & Triangular & 5873 & 17620 & & \\
\hline Unit cost of convictions & 20,362 & Triangular & 10181 & 30543 & & \\
\hline $\begin{array}{l}\text { Unit cost for sex offenders treatment } \\
\text { programmes }\end{array}$ & 11,025 & Triangular & 5513 & 16538 & & \\
\hline $\begin{array}{l}\text { Proportion of cases ending in court } \\
\text { proceedings }\end{array}$ & 0.176 & Beta & 0 & 1 & 4100 & 19200 \\
\hline Proportion of cases ending in convictions & 0.107 & Beta & 0 & 1 & 2500 & 20800 \\
\hline $\begin{array}{l}\text { Proportion of cases of sexual abuse ending } \\
\text { in sex offenders treatment programmes }\end{array}$ & 0.048 & Beta & 0 & 1 & 1118 & 22182 \\
\hline \multicolumn{7}{|l|}{ Social care costs } \\
\hline Foster care cost per day & 88 & Triangular & 44 & 132 & & \\
\hline Local Authority care cost per day & 455 & Triangular & 228 & 683 & & \\
\hline Unit cost of Child Protection Plan & 5,321 & Triangular & 2661 & 7982 & & \\
\hline Monthly child care support cost & 328 & Triangular & 164 & 492 & & \\
\hline $\begin{array}{l}\text { Proportion newly looked after children } \\
\text { receiving intervention in foster care }\end{array}$ & 0.777 & Beta & 0 & 1 & 24150 & 6920 \\
\hline $\begin{array}{l}\text { Proportion newly looked after children } \\
\text { receiving intervention in Local Authority care }\end{array}$ & 0.139 & Beta & 0 & 1 & 4340 & 26730 \\
\hline
\end{tabular}


Table 27. continued

\begin{tabular}{|c|c|c|c|c|c|c|c|}
\hline & $\begin{array}{l}\text { Central } \\
\text { value }\end{array}$ & SE & Distribution & $\begin{array}{l}\text { Lower } \\
\text { limit }\end{array}$ & $\begin{array}{l}\text { Upper } \\
\text { limit }\end{array}$ & $\alpha$ & $\beta$ \\
\hline \multicolumn{8}{|l|}{ Special education costs } \\
\hline $\begin{array}{l}\text { Unit cost of special educational support per } \\
\text { victim }\end{array}$ & 3,740 & & Triangular & 1870 & 5610 & & \\
\hline $\begin{array}{l}\text { Marginal effect of child maltreatment on } \\
\text { receipt of special education support }\end{array}$ & 0.22 & & Beta & 0 & 1 & 34 & 47 \\
\hline \multicolumn{8}{|l|}{ Long-term health-related costs } \\
\hline \multicolumn{8}{|l|}{ Annual cost of disorders } \\
\hline Depression (NHS costs only) & 2,417 & & Triangular & 1209 & 3627 & & \\
\hline Depression (productivity costs) & 4,478 & & Triangular & 2239 & 6718 & & \\
\hline Anxiety (NHS costs only) & 1,282 & & Triangular & 641 & 1923 & & \\
\hline Anxiety (productivity costs) & 662 & & Triangular & 331 & 992 & & \\
\hline Smoking (NHS and productivity costs) & 1,805 & & Triangular & 902 & 2707 & & \\
\hline Smoking (NHS costs only) & 430 & & Triangular & 215 & 645 & & \\
\hline Alcohol abuse (NHS costs only) & 211 & & Triangular & 106 & 317 & & \\
\hline Alcohol abuse (criminal justice costs) & 1,822 & & Triangular & 911 & 2734 & & \\
\hline Alcohol abuse (productivity costs) & 529 & & Triangular & 265 & 794 & & \\
\hline \multicolumn{8}{|l|}{$\begin{array}{l}\text { Marginal effect of child maltreatment on } \\
\text { probability of disorders }\end{array}$} \\
\hline Depression at age 33 & 0.053 & 0.013 & Gamma & & & 17.14 & 0.00 \\
\hline Depression at age 42 & 0.078 & 0.014 & Gamma & & & 29.19 & 0.00 \\
\hline Depression at age 50 & 0.041 & 0.014 & Gamma & & & 8.27 & 0.00 \\
\hline Depression at age 55 & 0.052 & 0.014 & Gamma & & & 14.20 & 0.00 \\
\hline \multicolumn{8}{|l|}{ Anxiety } \\
\hline Anxiety at age 33 & 0.026 & 0.009 & Gamma & & & 8.63 & 0.00 \\
\hline Anxiety at age 42 & 0.042 & 0.010 & Gamma & & & 16.54 & 0.00 \\
\hline Anxiety at age 50 & 0.035 & 0.011 & Gamma & & & 10.14 & 0.00 \\
\hline Anxiety at age 55 & 0.034 & 0.011 & Gamma & & & 9.06 & 0.00 \\
\hline \multicolumn{8}{|l|}{ Smoking } \\
\hline Current smoking at age 33 & 0.034 & 0.016 & Gamma & & & 4.64 & 0.01 \\
\hline Current smoking at age 42 & 0.034 & 0.014 & Gamma & & & 5.60 & 0.01 \\
\hline Current smoking at age 50 & 0.032 & 0.014 & Gamma & & & 5.45 & 0.01 \\
\hline \multicolumn{8}{|l|}{ Alcohol } \\
\hline Heavy drinking at age 50 or more & 0.029 & 0.009 & Gamma & & & 9.57 & 0.00 \\
\hline \multicolumn{8}{|l|}{ Reduced employment } \\
\hline \multicolumn{8}{|l|}{$\begin{array}{l}\text { Marginal effect of child maltreatment on } \\
\text { probability of being employed }\end{array}$} \\
\hline Employed at age 42 & -0.027 & 0.011 & Gamma & & & 5.67 & 0.00 \\
\hline Employed at age 50 & -0.036 & 0.012 & Gamma & & & 8.42 & 0.00 \\
\hline
\end{tabular}

All costs are in 2015 UKE. SE = standard error. $M V R=$ maltreatment or violence-related. 
Table 28. Discounted lifetime costs per victim of non-fatal child maltreatment: central estimate

\begin{tabular}{|c|c|c|c|}
\hline & Value $(£)$ & 95\% Uncertainty Interval & $\%$ Total \\
\hline Unplanned hospital admissions for injuries & 120 & $(83,141)$ & $<1$ \\
\hline Short-term mental health problems & 18,553 & $(9,758,29,833)$ & 21 \\
\hline Short-term health-related costs & 18,673 & $(9,841,29,974)$ & 21 \\
\hline Anxiety & 954 & $(311,2,094)$ & 1 \\
\hline Depression & 5,145 & $(1,782,10,740)$ & 6 \\
\hline Smoking & 528 & $(100,1,461)$ & 1 \\
\hline Alcohol abuse & 537 & $(148,1,262)$ & 1 \\
\hline Long-term health-related costs & 7,164 & $(2,341,15,558)$ & 8 \\
\hline Criminal justice system costs incurred by perpetrators & 4,316 & $(2,509,6,165)$ & 5 \\
\hline Social care costs & 38,132 & $(22,679,53,346)$ & 43 \\
\hline Special education costs & 7,068 & $(2,162,14,455)$ & 8 \\
\hline Reduced employment & 14,037 & $(5,364,26,010)$ & 16 \\
\hline Total & 89,390 & $(44,896,145,508)$ & 100 \\
\hline
\end{tabular}

All costs are discounted and in 2015 UKE. 
Figure 1. Discounted lifetime costs per victim of non-fatal child maltreatment: distribution of values from probabilistic sensitivity analysis.

(a) Frequency distribution

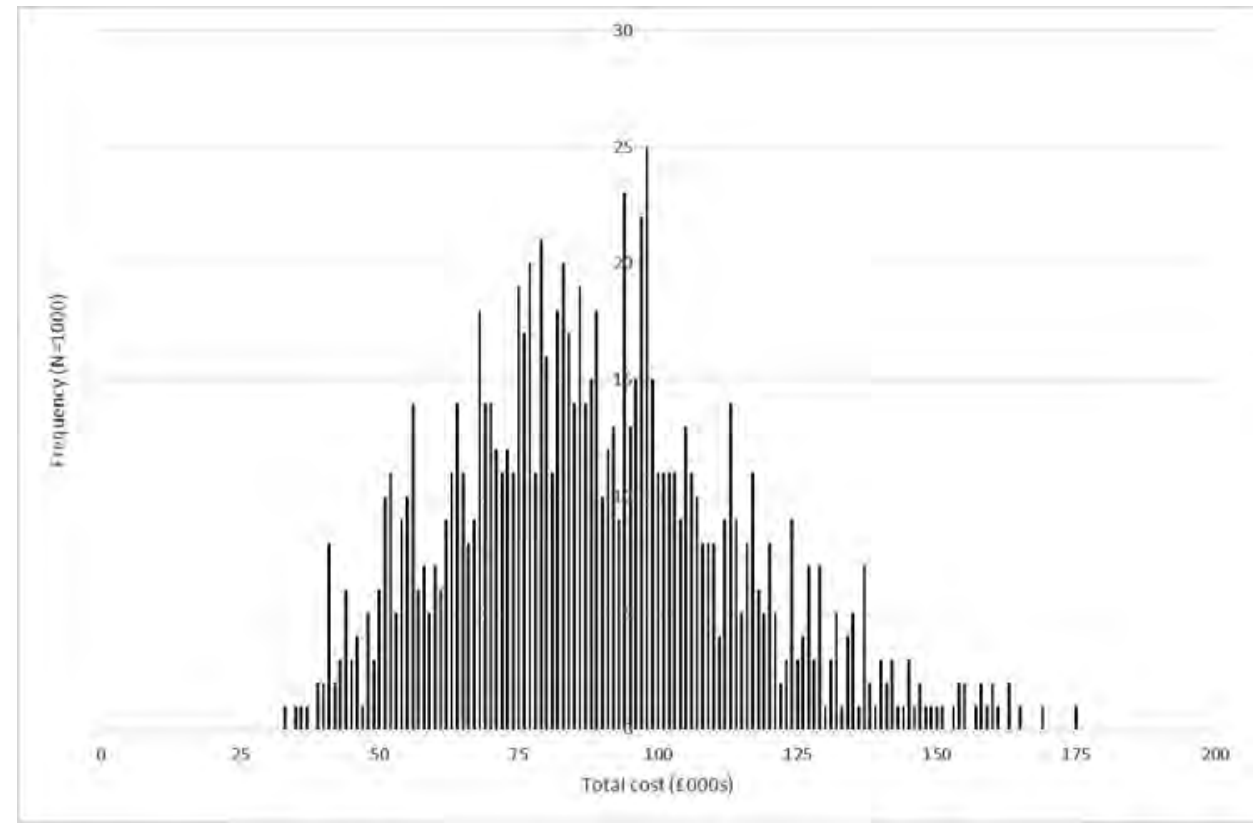

(b) Probability values are higher than level

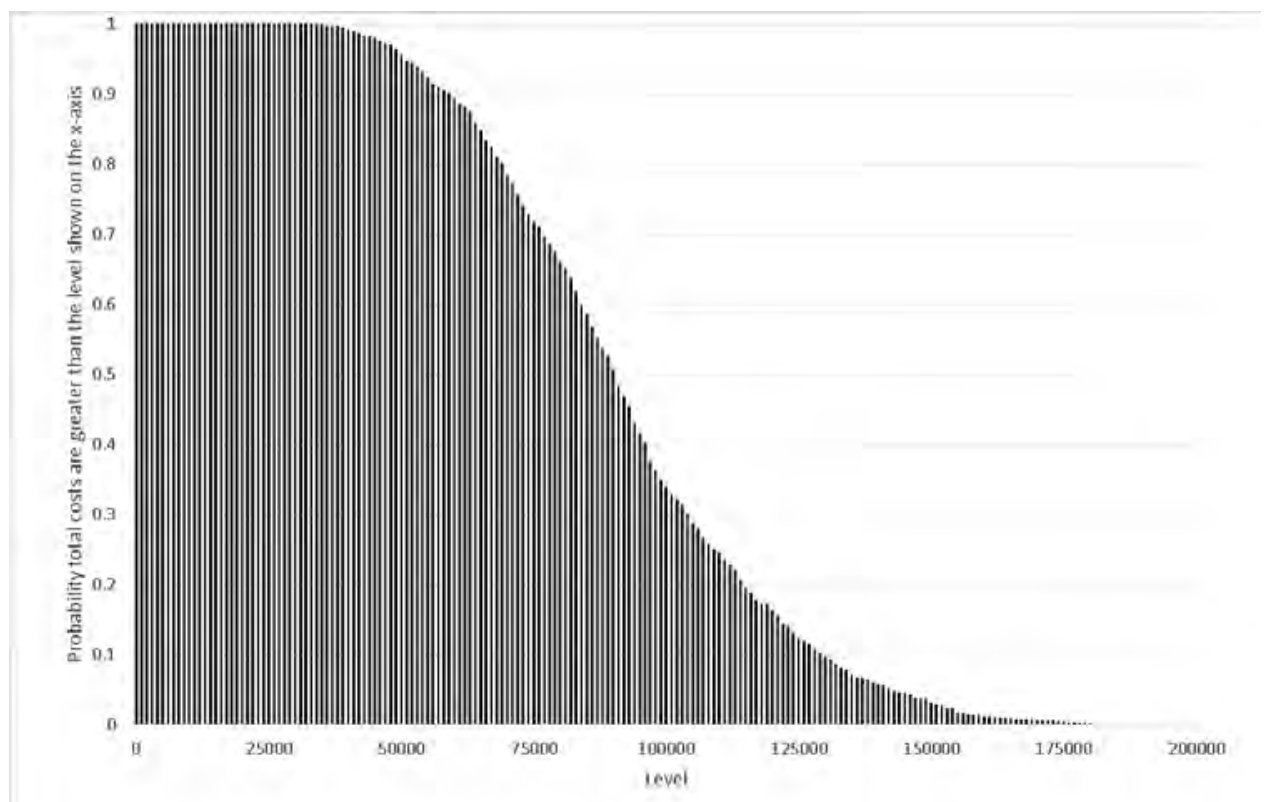




\subsection{Results}

\section{Lifetime costs per victim of non-fatal child maltreatment}

Table 28 presents central estimates of the discounted average lifetime cost of non-fatal child maltreatment per victim in 2015 UKE. We estimate the total cost to be $£ 89,390$ (95\% uncertainty interval $£ 44,896$ to $£ 145,508$ ). The largest contributors to these costs were social care costs (costs per victim $£ 38,132 ; 43 \%$ of the total), short-term health-related costs ( $£ 18,673$; $21 \%)$ and reduced employment costs ( $£ 14,037$; $16 \%$ ). The uncertainty intervals are wide, reflecting the uncertainty in the parameters included in the calculations. The distribution of values from the PSA are shown in Figure 1 (a). There is a 96\% probability that the total cost is greater than $£ 50,000$, a $34 \%$ probability it is greater than $£ 100,000$, and a $3 \%$ probability it is greater than $£ 150,000$ (Figure $1(b)$ ).

The scenarios evaluated in the deterministic sensitivity analysis, and the impact on total costs, are shown in Table 29. Discounted lifetime costs per victim of non-fatal child maltreatment were sensitive to the choice of discount rate, but we used the values recommended by HM Treasury for the central estimate. They were not sensitive to changes in assumptions surrounding hospital admissions for injuries, but were sensitive to the marginal effects of child maltreatment on mental health problems in the short-term, about which there is uncertainty. Total costs varied substantially when the marginal effects of child maltreatment on long-term healthrelated outcomes were increased, but not when the ages affected was increased. Given the source of the marginal effects, we consider the central estimates for this cost component to be robust; we also note the costs do not vary much when the costs from Fang et al were used instead, indicating the costs were similar in the two studies. Total costs were sensitive to changes in assumptions around social care costs, but not special education costs. They were also sensitive to assumptions about the costs associated with reduced employment: while the marginal effects were generated from our analysis of NCDS data it is notable that when costs from Fang et al were applied the total lifetime costs increased by over $60 \%$ to $£ 150,000$. 
Table 29. Discounted lifetime costs per victim of non-fatal child maltreatment: univariate deterministic sensitivity analysis

\begin{tabular}{|c|c|c|}
\hline & & Value $(£)$ \\
\hline Central estimate & & 89,390 \\
\hline Central estimate assumption & Changed to (all else equal) & \\
\hline \multicolumn{3}{|l|}{ Discount rate } \\
\hline $\begin{array}{l}\text { Years } 0 \text { to } 30=3.5 \% \text { per annum; years } 31 \text { to } 75=3 \% \text { per } \\
\text { annum; years } 76 \text { to } 125=2.5 \% \text { per annum }\end{array}$ & All years $=0 \%$ per annum & 148,747 \\
\hline $\begin{array}{l}\text { Years } 0 \text { to } 30=3.5 \% \text { per annum; years } 31 \text { to } 75=3 \% \text { per } \\
\text { annum; years } 76 \text { to } 125=2.5 \% \text { per annum }\end{array}$ & All years $=5 \%$ per annum & 75,884 \\
\hline \multicolumn{3}{|l|}{ Unplanned hospital admissions for injuries } \\
\hline 8,685 unplanned admissions across all victims & $\begin{array}{l}\text { Each victim has one unplanned admission; } 31.2 \% \\
\text { have a second admission }\end{array}$ & 90,389 \\
\hline 8,685 unplanned admissions across all victims & $\begin{array}{l}\text { Each victim has one unplanned admission; } 31.2 \% \\
\text { have a second admission; each admission is } \\
\text { associated with } 11.5 \text { outpatient visits and } 11.5 \mathrm{GP} \\
\text { visits }\end{array}$ & 92,758 \\
\hline \multicolumn{3}{|l|}{ Short-term mental health problems } \\
\hline $\begin{array}{l}\text { Annual cost per victim with mental health disorder }=£ 1,351 \text { to } \\
£ 3605 \text { depending on disorder }\end{array}$ & $\begin{array}{l}\text { Annual cost per victim with mental health disorder } \\
\text { doubles }\end{array}$ & 100,843 \\
\hline $\begin{array}{l}\text { Marginal effect of child maltreatment on probability of mental } \\
\text { health problems }=+0.061 \text { to }+0.345 \text { depending on age and type } \\
\text { of disorder }\end{array}$ & $\begin{array}{l}\text { Marginal effect of child maltreatment on } \\
\text { probability of mental health problems }=+0.05\end{array}$ & 75,657 \\
\hline $\begin{array}{l}\text { Marginal effect of child maltreatment on probability of mental } \\
\text { health problems }=+0.061 \text { to }+0.345 \text { depending on age and type } \\
\text { of disorder }\end{array}$ & $\begin{array}{l}\text { Marginal effect of child maltreatment on } \\
\text { probability of mental health problems }=+0.5\end{array}$ & 119,035 \\
\hline $\begin{array}{l}\text { Annual criminal justice costs for children with conduct disorder } \\
=£ 3,436\end{array}$ & $\begin{array}{l}\text { Annual criminal justice costs for children with } \\
\text { conduct disorder doubles }\end{array}$ & 96,490 \\
\hline \multicolumn{3}{|l|}{ Short-term health-related costs } \\
\hline Total short-term costs as in base case & $\begin{array}{l}\text { Total short-term health care costs as in Fang et al., } \\
\text { updated to } 2014 / 15 \cup K £(=£ 24,167)\end{array}$ & 94,884 \\
\hline \multicolumn{3}{|l|}{ Long-term health-related costs } \\
\hline $\begin{array}{l}\text { Marginal effect of child maltreatment on all types of long-term } \\
\text { health care costs }=+0.029 \text { to }+0.077 \text { in effective age ranges } \\
\text { depending on age and condition }\end{array}$ & $\begin{array}{l}\text { Marginal effect of child maltreatment on all } \\
\text { conditions and ages (maintaining original effective } \\
\text { age ranges) }=+0.1\end{array}$ & 97,571 \\
\hline $\begin{array}{l}\text { Marginal effect of child maltreatment on all types of long-term } \\
\text { health care costs }=+0.029 \text { to }+0.077 \text { in effective age ranges } \\
\text { depending on age and condition }\end{array}$ & $\begin{array}{l}\text { Marginal effect of child maltreatment on all } \\
\text { conditions and ages (maintaining original effective } \\
\text { age ranges) }=+0.2\end{array}$ & 112,916 \\
\hline $\begin{array}{l}\text { Marginal effect of child maltreatment on all types of long-term } \\
\text { health care costs }=+0.029 \text { to }+0.077 \text { in effective age ranges } \\
\text { depending on age and condition }\end{array}$ & $\begin{array}{l}\text { Marginal effects of child maltreatment at youngest } \\
\text { effective age extrapolated to } 18 \text {, and at oldest } \\
\text { effective age extrapolated to } 100\end{array}$ & 93,469 \\
\hline Total long-term costs as in base case & $\begin{array}{l}\text { Total long-term health care costs as in Fang et al., } \\
\text { updated to } 2014 / 15 \text { UK£ }(=£ 7,795)\end{array}$ & 90,021 \\
\hline
\end{tabular}


Table 29. continued

\begin{tabular}{|c|c|c|}
\hline & & Value $(f)$ \\
\hline \multicolumn{3}{|l|}{ Criminal justice system costs incurred by perpetrators } \\
\hline $\begin{array}{l}17.6 \% \text { cases end in court proceedings and } 10.7 \% \text { end in } \\
\text { convictions }\end{array}$ & All cases end in court proceedings and convictions & 115,045 \\
\hline Criminal justice costs as in base case & $\begin{array}{l}\text { Criminal justice cost as in Fang et al., updated to } \\
2014 / 15 \text { UKE }(=£ 3,533)\end{array}$ & 88,607 \\
\hline \multicolumn{3}{|l|}{ Social care costs } \\
\hline $\begin{array}{l}\text { Unit cost of child protection plan }=£ 5,321 \text {; monthly child care } \\
\text { support cost }=£ 328\end{array}$ & $\begin{array}{l}\text { Unit cost of child protection plans and monthly } \\
\text { child care support costs double }\end{array}$ & 96,900 \\
\hline Child social care costs as in base case & $\begin{array}{l}\text { Child social care costs as in Fang et al., updated to } \\
2014 / 15 \text { UKE }(=£ 5,721)\end{array}$ & 56,979 \\
\hline \multicolumn{3}{|l|}{ Special education costs } \\
\hline $\begin{array}{l}\text { Unit cost of special educational support per victim }=£ 3,740 \text { per } \\
\text { annum }\end{array}$ & $\begin{array}{l}\text { Unit cost of special educational needs provision } \\
\text { doubles }\end{array}$ & 96,459 \\
\hline $\begin{array}{l}\text { Marginal effect of child maltreatment on receipt of special } \\
\text { education support }=0.22\end{array}$ & $\begin{array}{l}\text { Marginal effect of child maltreatment on receipt of } \\
\text { special education support }=0.11\end{array}$ & 85,863 \\
\hline $\begin{array}{l}\text { Marginal effect of child maltreatment on receipt of special } \\
\text { education support }=0.22\end{array}$ & $\begin{array}{l}\text { Marginal effect of child maltreatment on receipt of } \\
\text { special education support }=0.33\end{array}$ & 92,946 \\
\hline \multicolumn{3}{|l|}{ Reduced employment } \\
\hline Earnings if employed = national average earnings by age group & $\begin{array}{l}\text { Earnings if employed }=75 \% \text { of the national average } \\
\text { earnings by age group }\end{array}$ & 85,881 \\
\hline Earnings if employed = national average earnings by age group & $\begin{array}{l}\text { Earnings if employed }=50 \% \text { of the national average } \\
\text { earnings by age group }\end{array}$ & 82,372 \\
\hline Annual increase in wages $2 \%$ & Annual increase in wages $1 \%$ & 84,876 \\
\hline Annual increase in wages $2 \%$ & Annual increase in wages $3 \%$ & 96,008 \\
\hline $\begin{array}{l}\text { Marginal effect of child maltreatment on not being employed } \\
=0 \text { at age } 33,-0.027 \text { at age } 42,-0.035 \text { at age } 50 \text { and } 0 \text { at age } \\
55 \text {, with linear interpolation }\end{array}$ & $\begin{array}{l}\text { Marginal effect of child maltreatment on not being } \\
\text { employed at ages } 42 \text { and } 50=-0.05 \text {, all else equal }\end{array}$ & 98,349 \\
\hline $\begin{array}{l}\text { Marginal effect of child maltreatment on not being employed } \\
=0 \text { at age } 33,-0.027 \text { at age } 42,-0.035 \text { at age } 50 \text { and } 0 \text { at age } \\
55 \text {, with linear interpolation }\end{array}$ & $\begin{array}{l}\text { Marginal effect of child maltreatment on not being } \\
\text { employed at ages } 42 \text { and } 50=-0.1 \text {, all else equal }\end{array}$ & 121,344 \\
\hline $\begin{array}{l}\text { Marginal effect of child maltreatment on not being employed } \\
=0 \text { at age } 33,-0.027 \text { at age } 42,-0.035 \text { at age } 50 \text { and } 0 \text { at age } \\
55 \text {, with linear interpolation }\end{array}$ & $\begin{array}{l}\text { Marginal effect of child maltreatment on not being } \\
\text { employed at ages } 42 \text { and } 50=-0.2 \text {, all else equal }\end{array}$ & 167,334 \\
\hline $\begin{array}{l}\text { Marginal effect of child maltreatment on not being employed } \\
=0 \text { at age } 33,-0.027 \text { at age } 42,-0.035 \text { at age } 50 \text { and } 0 \text { at age } \\
55 \text {, with linear interpolation }\end{array}$ & $\begin{array}{l}\text { Marginal effects of child maltreatment at age } 42 \\
(=-0.027) \text { extended to age } 18, \text { and at age } 50(= \\
-0.035) \text { extended to age } 67\end{array}$ & 113,515 \\
\hline Cost of reduced employment as in base case & $\begin{array}{l}\text { Cost of reduced employed as in Fang et al., updated } \\
\text { to } 2014 / 15 \cup K £(=£ 4,360 \text { per annum from ages } \\
18-64 \text { inclusive, } £ 0 \text { otherwise })\end{array}$ & 150,001 \\
\hline
\end{tabular}

All costs are in 2015 UKE and discounted unless indicated otherwise. GP = general practitioner 


\section{Lifetime costs per death from child maltreatment}

Our central estimate of the discounted lifetime cost per death from child maltreatment was $£ 940,758$, comprising $£ 5,408$ health care costs ( $1 \%$ of the total) and $£ 935,350$ lost productivity costs (99\%; Table 30). The results were sensitive to the discount rate and assumptions concerning lost productivity over the lifetime (Table 31).

Table 30. Discounted lifetime costs per death from child maltreatment: central estimate

\begin{tabular}{lcc}
\hline & Value (£) & \% Total \\
\hline Health care costs & 5,408 & 1 \\
\hline Lost productivity & 935,350 & 99 \\
\hline Total & 940,758 & 100 \\
\hline
\end{tabular}

All costs are discounted and in 2015 UKE.

Table 31. Discounted lifetime costs per death from child maltreatment: univariate deterministic sensitivity analysis

\begin{tabular}{|c|c|c|}
\hline & & Value ( $(£)$ \\
\hline Central estimate & & 940,758 \\
\hline Central estimate assumption & Changed to (all else equal) & \\
\hline \multicolumn{3}{|l|}{ Discount rate } \\
\hline $\begin{array}{l}\text { Years } 0 \text { to } 30=3.5 \% \text { per annum; years } 31 \text { to } 75=3 \% \text { per } \\
\text { annum; years } 76 \text { to } 125=2.5 \% \text { per annum }\end{array}$ & All years $=0 \%$ per annum & $2,994,842$ \\
\hline $\begin{array}{l}\text { Years } 0 \text { to } 30=3.5 \% \text { per annum; years } 31 \text { to } 75=3 \% \text { per } \\
\text { annum; years } 76 \text { to } 125=2.5 \% \text { per annum }\end{array}$ & All years $=5 \%$ per annum & 504,483 \\
\hline \multicolumn{3}{|l|}{ Health care costs } \\
\hline Health care costs associated with fatal injury $=£ 5,408$ & $\begin{array}{l}\text { Health care costs associated with fatal injury = } \\
£ 13,863\end{array}$ & 949,213 \\
\hline \multicolumn{3}{|l|}{ Lost productivity } \\
\hline $\begin{array}{l}\text { Earnings if employed = national average earnings by age } \\
\text { group }\end{array}$ & $\begin{array}{l}\text { Earnings if employed }=75 \% \text { of the national average } \\
\text { earnings by age group }\end{array}$ & 706,921 \\
\hline $\begin{array}{l}\text { Earnings if employed = national average earnings by age } \\
\text { group }\end{array}$ & $\begin{array}{l}\text { Earnings if employed }=50 \% \text { of the national average } \\
\text { earnings by age group }\end{array}$ & 473,083 \\
\hline Employment rate $=$ national average rates by age group & $\begin{array}{l}\text { Employment rate }=75 \% \text { of the national average rates } \\
\text { by age group }\end{array}$ & 706,921 \\
\hline Employment rate $=$ national average rates by age group & $\begin{array}{l}\text { Employment rate }=50 \% \text { of the national average rates } \\
\text { by age group }\end{array}$ & 473,083 \\
\hline Annual increase in wages $=2 \%$ & Annual increase in wages $=1 \%$ & 663,419 \\
\hline Annual increase in wages $=2 \%$ & Annual increase in wages $=3 \%$ & $1,348,863$ \\
\hline \multicolumn{3}{|l|}{ Criminal justice system costs } \\
\hline Not included & $\begin{array}{l}\text { Assume all cases result in a court proceedings and a } \\
\text { conviction }\end{array}$ & 972,867 \\
\hline
\end{tabular}

All costs are discounted and in 2015 UKE. 


\section{Discussion}

\section{Summary of findings}

There is little evidence of the lifetime cost of child maltreatment in the UK. We used rich, representative UK data to estimate the effect of child maltreatment on a range of outcomes. We found that having experienced any form of child maltreatment was associated with worse mental health outcomes, smoking behaviour, alcohol use, lower probability of employment, and greater welfare dependence. We detected no robust effects of child maltreatment on physical health, heavy smoking and wages. Using these and other data we calculated that the discounted lifetime costs per victim of non-fatal child maltreatment in the UK were estimated as $£ 89,390$ per victim (95\% uncertainty interval $£ 44,896$ to $£ 145,508$ ). The discounted lifetime cost per death from child maltreatment in the UK was estimated to be $£ 940,758$. There was uncertainty in these estimates due to data limitations.

\section{Strengths and limitations}

This is the first UK-based study to calculate the lifetime costs of child maltreatment. A strength of our study is that we have included a wide range of cost components. Another is that several of these cost components - long-term healthrelated costs and productivity losses - are based on a detailed econometric analysis of the impact of child maltreatment using rich longitudinal data undertaken especially for this analysis. We undertook extensive sensitivity analysis to evaluate the impact of uncertainty in the analysis.

\section{Limitations resulting from the datasets}

Our analysis of the effect of child maltreatment was limited by the range of child maltreatment and outcome measures in the data. In addition, the structure of the datasets forced a reliance on the use of retrospective measures of child maltreatment, and precluded the possibility of exploiting within-family analyses to improve identification. The use of retrospective measures is a limitation as it relies on respondent recall and precludes cases of child maltreatment that resulted in the death of the child. Further, while we adjusted for a range of potential confounding factors, we cannot rule out the possibility that the effects of child maltreatment found in our analysis might be due to unobserved factors that are characteristics of families in which children are maltreated (e.g. other measures of adversity not included in our data).

We included numerous effects associated with child maltreatment in our analysis. The effects we initially analysed were guided by the literature review. In our analysis of the NCDS and the ELSA, we did not find any effects for obesity, hypertension, type 2 diabetes, raised cholesterol, cancer and we therefore did not include them in the cost analysis. Some other effects found to be important in previous studies we were available in our data (e.g., days off work, premature mortality, drug use) so we did not analyse them.

\section{Limitations of the cost analysis}

The cost analysis has several limitations, mainly arising from shortcomings in the data. First and foremost data were not available for many of the probabilities and costs used to produce the estimates in this study. We had to make many assumptions using available data. As highlighted in the results section, discounted lifetime costs per victim of non-fatal child maltreatment were especially sensitive to the impact of child maltreatment on short-term health-related costs, especially mental health disorders, and there was uncertainty surrounding these impacts. For several of the other outcomes, there was uncertainty in the analysis parameters but this did not seem to affect lifetime costs appreciably, or when the parameters were varied this did affect lifetime costs, but there was less uncertainty in the values used in the central estimate.

For several of the cost components (e.g. unplanned hospital admissions for injuries, criminal justice system costs) we required longitudinal data on the number of events for new cases of child maltreatment in a single year to be able to 
accurately calculate incidence-based costs. These data were not available so we were required to make 'steady-state' assumptions, as have been used by other researchers. This assumption requires that the number of events remains fairly constant over time, which may not hold for the cost components considered here. The cost components where we made this assumption account for a relatively small proportion of the total cost.

\section{Comparison with other studies}

As evidenced by our literature reviews, there have been a range of findings across studies evaluating the effect of child maltreatment, showing that different types of abuse have a range of effect sizes on different outcomes. We detected no robust effects of child maltreatment on physical health, heavy smoking and wages. These results are not consistent with some studies, though it is difficult to make comparisons between studies as they are fraught with difficulties due to differences in study populations, sampling methods and variable definitions (especially child maltreatment measures)

In terms of the cost analysis this is the first study to measure the lifetime costs of child maltreatment in the UK so comparisons with UK studies are not possible (though there is UK evidence that child maltreatment is associated with poor socioeconomic outcomes in adulthood ${ }^{30}$ ). Fang and colleagues (Fang, Brown et al. 2012) estimated the lifetime costs of child maltreatment in the USA and our study used a similar approach. Comparisons between countries are difficult due to differences in health care, social care, education and criminal justice systems, and also costs. Comparisons between the two studies are made more difficult given differences in methodology, especially in how costs were classified between different categories, and in the choice of discount rate. Bearing this in mind the lifetime costs per death from child maltreatment are broadly comparable between the two studies (our estimate was $£ 940,758$, versus US\$1,272,900 in Fang et al.). The lifetime costs per victim of non-fatal child maltreatment are noticeably different in that our total costs are substantially lower ( $£ 89,930$ versus US\$210,012). Looking at the costs by component the main difference between the two studies is in terms of the productivity losses. Partly this is because in our study these only include the effects of reduced employment as other elements are partly included in the long-term health-related costs. However, as seen in our univariate deterministic sensitivity analysis when the productivity losses from Fang et al. are updated to 2015 prices and converted into UKE and included in our estimates our total lifetime costs increased considerably ( $£ 150,001)$.

\section{Future research}

Our study highlights that further research would be beneficial on several aspects. First, our quantitative analysis of the effect of child maltreatment was mainly related to maltreatment by primary caregivers; further research accounting for maltreatment by other parties would be useful. Second, further research would be beneficial to evaluate separately the costs of different types of child maltreatment (e.g. physical, emotional and sexual above, neglect), although this is unlikely to be straightforward. Third, as indicated in the limitations section, our analysis has been limited by availability of data; repeating the analysis in future as better data become available would be informative. Fourth, while our study has highlighted the lifetime costs associated with child maltreatment are large, this does not mean that interventions to prevent child maltreatment are necessarily cost-effective - to judge this, the costs of interventions need to be balanced against the costs savings they produce and their health and other benefits. Hence, further research would be beneficial to evaluate the cost-effectiveness of interventions to reduce child maltreatment.

30 See, for example the recent paper by Pinto Pereira and colleagues (Pinto Pereira, S.M., L. Li, C. Power (2017). Child maltreatment and adult living standards at 50 years. Pediatrics 139(1):e20161595). 


\section{Implications of this research}

1. The lifetime costs of non-fatal and fatal child maltreatment in the UK are substantial. Our findings indicate how much money UK society is spending on a case of child maltreatment, and by implication, the amount that would be saved if child maltreatment was prevented.

2. This research, focusing on the lifetime costs of child maltreatment, can be used in economic evaluations of child maltreatment intervention/ prevention activities to quantify the costs saved from reducing the number of children who are maltreated. It is important to remember that this research focuses on the costs of maltreatment from a parent/primary care-giver due to the limitations of the data sets that have been outlined. That means that thought and care is needed when applying this research to areas where the maltreatment may be perpetrated by someone who isn't the primary care-giver. For example, it doesn't follow that social care costs would necessarily result from such a case of child maltreatment as the child may not go into care.
3. Articulating and quantifying the longer-term economic impacts of child maltreatment can help develop and shape a wider policy narrative about child maltreatment. Our research identifies the different components of the lifetime costs of child maltreatment and the size of the contribution of each sector in society. For example, child protection is often seen as an issue that is for children's social services to tackle. This research shows very clear the significant costs that child maltreatment has on the economy more generally, for example through impacts on productivity and through the costs that are created for health services. 
Barnett, A., H. Birnbaum, P. Y. Cremieux, A. M. Fendrick and M. Slavin (2000). "The costs of cancer to a major employer in the United States: A casecontrol analysis." American Journal of Managed Care 6(11): 1243-1251.

Birnbaum, H., S. Leong and A. Kabra (2003). "Lifetime medical costs for women: Cardiovascular disease, diabetes, and stress urinary incontinence." Womens Health Issues 13(6): 204-213.

Boschung, M., Kendrick, A., Addy, S. (2015). The Cost of Child Maltreatment to the Alabama Economy in 2013. Alabama The University of Alabama.

Briggs, A., Sculpher, M., Claxton, K. (2006). Decision modelling for health economic evaluation. . Oxford, Oxford University Press.

Brown, D. S., X. Fang and C. S. Florence (2011).

"Medical costs attributable to child maltreatment a systematic review of short- and long-term effects." Am J Prev Med 41(6): 627-635.

Cabinet Office (2003). Alcohol misuse: how much does it cost? London Cabinet Office

Choi, B. C., L. Robson and E. Single (1997).

"Estimating the economic costs of the abuse of tobacco, alcohol and illicit drugs: a review of methodologies and Canadian data sources." Chronic Dis Can 18(4): 149-165.

Christensen, M. C., T. G. Nielsen, S. Ridley, F. E. Lecky and S. Morris (2008). "Outcomes and costs of penetrating trauma injury in England and Wales." Injury 39(9): 1013-1025.

Christensen, M. C., S. Ridley, F. E. Lecky, V. Munro and S. Morris (2008). "Outcomes and costs of blunt trauma in England and Wales." Crit Care 12(1): R23.

Corso, P. S. and A. R. Fertig (2010). "The economic impact of child maltreatment in the United States: are the estimates credible?" Child Abuse Negl 34(5): 296-304.

DCSF January Schools Census. (2009). "Budgeted net expenditure on the education of children with special educational needs." from https:// www.theyworkforyou.com/wrans/?id=2009-0331c.266343.h.

Department for Education (DfE) (2015). Children looked after in England (including adoption and care leavers) year ending 31 March 2015. London. SFR 34/2015.
Department for Education (DfE) (2016). "Children in need statistics. Additional data provided by the DfE with the NSPCC."

DOH (2015). National Schedule of Reference Costs 2014 to 2015. D. o. Health. London.

Fang, X., D. S. Brown, C. S. Florence and J. A. Mercy (2012). "The economic burden of child maltreatment in the United States and implications for prevention." Child Abuse Negl 36(2): 156-165.

Fineberg, N. A., P. M. Haddad, L. Carpenter, B. Gannon, R. Sharpe, A. H. Young, E. Joyce, J. Rowe, D. Wellsted, D. J. Nutt and B. J. Sahakian (2013). "The size, burden and cost of disorders of the brain in the UK." J Psychopharmacol 27(9): 761-770.

Fisher, H. (2016). Unpublished work

Florence, C., D. S. Brown, X. Fang and H. F. Thompson (2013). "Health care costs associated with child maltreatment: impact on medicaid." Pediatrics 132(2): 312-318.

Gonzalez-Izquierdo, A., M. Cortina-Borja, J. Woodman, J. Mok, J. McGhee, J. Taylor, C. Parkin and R. Gilbert (2014). "Maltreatment or violence-related injury in children and adolescents admitted to the NHS: comparison of trends in England and Scotland between 2005 and 2011." Bmj Open 4(4).

Hennes, H., Kini, N, Palusci, V. (2001). "The epidemiology, clinical characteristics and public health implications of shaken baby syndrome." Journal of Aggression, maltreatment and trauma 5(1): 19-40.

Hodgson, T. A. and M. R. Meiners (1982). "Cost-ofIIIness Methodology - a Guide to Current Practices and Procedures." Milbank Memorial Fund QuarterlyHealth and Society 60(3): 429-462.

Luce, B., W. Manning, J. Siegel and J. Lipscomb (1996). Estimating Costs in Cost-Effectiveness Analysis. Cost-effectiveness in Health and Medicine. M. Gold, Siegel, JE, Russell, LB, . New York, Oxford University Press.

McCrone, P., Dhanasiri, S., Patel, A., Knapp, M., Lawton-Smith, S. (2008). Paying the Price - The cost of metnal heatlh care in Engand to 2026. London, King's Fund.

Meltzer, H., R. Gatward, T. Corbin, R. Goodman and T. Ford (2003). The mental health of young people looked after by local authorities in England. London, Office for National Statistics. 
Minnis, H., K. Everett, A. J. Pelosi, J. Dunn and M. Knapp (2006). "Children in foster care: mental health, service use and costs." Eur Child Adolesc Psychiatry 15(2): 63-70.

National Institute for Health and Care Excellence, N. (2013). Guide to the methods of technology appraisal London.

Official Statistics (2016). Regional labour market statistics: X01 Regional employment by age.

September 2016

ONS (2014). Distribution of median and mean income and tax by age range and gender, 2013-14.

Peterson, C., L. K. Xu, C. Florence, S. E. Parks, T. R. Miller, R. G. Barr, M. Barr and R. Steinbeigle (2014). "The Medical Cost of Abusive Head Trauma in the United States." Pediatrics 134(1): 91-99.

Reckoner, R. (2016). The local cost of tobacco, Action on Smoking and Health

Rutter, M., Giller, H. and Hagell, A. (1998). Anti-social Behaviour by Young People. Cambridge, Cambridge University Press.

Saied-Tessier, A. (2014). Estimating the costs of child sexual abuse in the UK London, NSPCC.
Scott, S., M. Knapp, J. Henderson and B. Maughan (2001). "Financial cost of social exclusion: follow up study of antisocial children into adulthood." BMJ 323(7306): 191.

Snell, T., M. Knapp, A. Healey, S. Guglani, S. EvansLacko, J. L. Fernandez, H. Meltzer and T. Ford (2013). "Economic impact of childhood psychiatric disorder on public sector services in Britain: estimates from national survey data." J Child Psychol Psychiatry 54(9): 977-985.

Summers, C. L. and E. M. Molyneux (1992).

"Suspected child abuse: cost in medical time and finance." Arch Dis Child 67(7): 905-908; discussion 908-910.

Thomas, C. a. M., S. (2000). "Cost of depression among adults in England in 2000." British Journal of Psychiatry 183: 514-519.

Wijlaars, L., Hardelid, P., Woodman, J., Allister, J., Cheung, R., Gilbert, R. (2016). "Who comes back with what: a retrospective database study on reasons for emergency readmission to hospital in children and young people in England." Arch Dis Child archdischild-2015-309290. Published Online First: 25 April 2016 


\section{Appendix}

\section{UK Studies of Child Maltreatment}

The following papers have been reviewed to perform the UK literature (in alphabetical order).

1. Arseneault, L., Cannon, M., Fisher, H. L., Polanczyk, G., Moffitt, T. E., \& Caspi, A. (2011). Childhood trauma and children's emerging psychotic symptoms: a genetically sensitive longitudinal cohort study. American Journal of Psychiatry, 168(1), 65-72.

2. Clark, C., Caldwell, T., Power, C., \& Stansfeld, S. A. (2010). Does the influence of childhood adversity on psychopathology persist across the lifecourse? A 45-year prospective epidemiologic study. Annals of epidemiology, 20(5), 385-394.

3. Collishaw, S., Dunn, J., O'connor, T. G., \& Golding, J. (2007). Maternal childhood abuse and offspring adjustment over time. Development and psychopathology, 19(02), 367-383.

4. Denholm, R., Power, C., \& Li, L. (2013). Adverse childhood experiences and child-to-adult height trajectories in the 1958 British birth cohort. International journal of epidemiology, 42(5), 1399-1409.

5. Denholm, R., Power, C., Thomas, C., \& Li, L. (2013). Child maltreatment and household dysfunction in a British birth cohort. Child abuse review, 22(5), 340-353.

6. Fisher, H. L., Caspi, A., Moffitt, T. E., Wertz, J., Gray, R., Newbury, J., ... \& Odgers, C. L. (2015). Measuring adolescents' exposure to victimization: The Environmental Risk (E-Risk) Longitudinal Twin Study. Development and psychopathology, 27(4pt2), 1399-1416.

7. Ford, E., Clark, C., \& Stansfeld, S. A. (2011). The influence of childhood adversity on social relations and mental health at mid-life. Journal of affective disorders, 133(1), 320-327.

8. Geoffroy, M. C., Pereira, S. P., Li, L., \& Power, C. (2016). Child neglect and maltreatment and childhood-to-adulthood cognition and mental health in a prospective birth cohort. Journal of the American Academy of Child \& Adolescent Psychiatry, 55(1), 33-40.
9. Jaffee, S. R., Caspi, A., Moffitt, T. E., Polo-Tomas, M., Price, T. S., \& Taylor, A. (2004). The limits of child effects: evidence for genetically mediated child effects on corporal punishment but not on physical maltreatment. Developmental psychology, 40(6), 1047.

10. Kelly-Irving, M., Lepage, B., Dedieu, D., Lacey, R., Cable, N., Bartley, M., ... \& Delpierre, C. (2013). Childhood adversity as a risk for cancer: findings from the 1958 British birth cohort study. BMC Public Health, 13(1), 1.

11. Li, L., Denholm, R., \& Power, C. (2014). Child maltreatment and household dysfunction: associations with pubertal development in a British birth cohort. International journal of epidemiology, dyu071.

12. Power, C., Pereira, S. M. P., \& Li, L. (2015). Childhood maltreatment and BMI trajectories to mid-adult life: follow-up to age 50y in a British birth cohort. PloS one, 10(3), e0119985.

13. Sidebotham, P., Golding, J., \& ALSPAC Study Team. (2001). Child maltreatment in the "Children of the Nineties": A longitudinal study of parental risk factors. Child abuse \& neglect, 25(9), 1177-1200.

14. Sidebotham, P., Heron, J., Golding, J., \& ALSPAC Study Team. (2002). Child maltreatment in the "Children of the Nineties:" deprivation, class, and social networks in a UK sample. Child abuse \& neglect, 26(12), 1243-1259.

15. Sidebotham, P., Heron, J., \& Teamc, T. A. S. (2003). Child maltreatment in the "children of the nineties:" the role of the child. Child abuse \& neglect, 27(3), 337-352.

16. Sidebotham, P., Heron, J., \& ALSPAC Study Team. (2006). Child maltreatment in the "children of the nineties": A cohort study of risk factors. Child abuse \& neglect, 30(5), 497-522.

17. Solís, C. B., Kelly-Irving, M., Fantin, R., Darnaudéry, M., Torrisani, J., Lang, T., \& Delpierre, C. (2015). Adverse childhood experiences and physiological wear-and-tear in midlife: Findings from the 1958 British birth cohort. Proceedings of the National Academy of Sciences, 112(7), E738-E746. 
18. Suderman, M., Borghol, N., Pappas, J. J., Pereira, S. M. P., Pembrey, M., Hertzman, C., ... \& Szyf, M. (2014). Childhood abuse is associated with methylation of multiple loci in adult DNA. BMC medical genomics, $7(1), 1$.

19. Thomas, C., Hyppönen, E., \& Power, C. (2008). Obesity and type 2 diabetes risk in midadult life: the role of childhood adversity. Pediatrics, 121(5), e1240-e1249.

20. Woodman, J., Freemantle, N., Allister, J., de Lusignan, S., Gilbert, R., \& Petersen, I. (2012). Variation in recorded child maltreatment concerns in UK primary care records: a cohort study using The Health Improvement Network (THIN) database. PLoS One, 7(11), e49808.

\section{Economics Studies of Child Maltreatment}

The following papers have been reviewed to perform the economics literature (in alphabetical order).

1. Bitler, M. P., \& Zavodny, M. (2004). Child maltreatment, abortion availability, and economic conditions. Review of Economics of the Household, 2(2), 119-141.

2. Currie, J., \& Tekin, E. (2012). Understanding the cycle childhood maltreatment and future crime. Journal of Human Resources, 47(2), 509-549.

3. Currie, J., \& Widom, C. S. (2010). Long-term consequences of child abuse and neglect on adult economic well-being. Child maltreatment, 15(2), 111-120.

4. Fletcher, J. M. (2009). Childhood mistreatment and adolescent and young adult depression. Social Science \& Medicine, 68(5), 799-806.

5. Fletcher, J., \& Schurer, S. (2015). Childhood Origins of Adulthood Noncognitive Skills: The Role of Chronic Health Problems and Exposure to Maltreatment (ARC lifecourse centre dp No. 2015-23).

6. Paxson, C., \& Waldfogel, J. (1999). Parental resources and child abuse and neglect. The American Economic Review, 89(2), 239-244.

7. Paxson, C., \& Waldfogel, J. (2002). Work, welfare, and child maltreatment. Journal of Labor Economics, 20(3), 435-474.
8. Paxson, C., \& Waldfogel, J. (2003). Welfare reforms, family resources, and child maltreatment. Journal of Policy Analysis and Management, 22(1), 85-113.

9. Reeve, R., \& Gool, K. (2013). Modelling the Relationship between Child Abuse and LongTerm Health Care Costs and Wellbeing: Results from an Australian Community-Based Survey. Economic Record, 89(286), 300-318.

\section{Cost Studies}

1. Peterson, C., Xu, L., Florence, C., Parks, S.E. (2015). Annual Cost of U.S. Hospital Visits for Pediatric Abusive Head Trauma. Child Maltreat. 20(3):162-9

2. Fang X1, Brown DS, Florence CS, Mercy JA. (2012).The economic burden of child maltreatment in the United Stated and implications for prevention. Child Abuse Negl. 36(2):156-65

3. Hennes, H., Kini, N. and Palusci, V.J.(2001). The epidemiology, clinical characteristics and public health implications of shaken baby syndrome. Journal of aggression, maltreatment and trauma, Vol.5, Iss.1

4. Wada, Ichiro, and Ataru Igarashi, (2014). The social costs of child abuse in Japan. Children and Youth Services Review 46 (2014): 72-77.

5. NSPCC (2011). The costs and consequences of child maltreatment: literature review for the NSPCC.

6. Wekerle, C. (2011). The dollars and senselessness in failing to prioritize childhood maltreatment prevention. Child abuse and neglect, Vol.35, Iss.3

7. Corso, P.S. and Fertig, A.R, (2010). The economic impact of child maltreatment in the United States: are the estimates credible?. Child abuse and neglect, Vol.34, Iss.5

8. Dolezal, T., McCollum, D. and Callahan, M. (2009). Hidden costs in health care: the economic impact of violence and abuse. Eden Prairie, Minnesota: Academy of Violence and Abuse

9. NICE, (2009). Costing statement: when to suspect child maltreatment. 
10. Alabama Children's Trust Fund et al.(2007). The costs of child abuse vs. child abuse prevention: Alabama's experience. University of Alabama. Center for Business and Economic Research

11. Wang, C. and Holton, J. (2007). Total estimated cost of child abuse and neglect in the United States. Chicago: Prevent Child Abuse America

12. World Health Organization. Violence and Injury Prevention Programme (2007). Preventing child maltreatment in Europe: a public health approach: policy briefing. Copenhagen: World Health Organization (WHO). Regional Office for Europe

13. Corso, P.S. and Lutzker, J.R. (2006). The need for economic analysis in research on child maltreatment. Child abuse and neglect, Vol.30, Iss.7

14. Giardino, A.P. et al. (1995). Funding realities: child abuse diagnostic evaluations in the health care setting. Child abuse and neglect, Vol.23, Iss.6

15. Irazuzta, J.E. et al. (1997). Outcome and cost of child abuse. Child abuse and neglect, Vol.21, Iss. 8

16. Dubowitz, H (1990). Costs and effectiveness of interventions in child maltreatment. Child abuse and neglect, Vol.14, Iss.2

17. Summers, C. L., and E. M. Molyneux. (1992). Suspected child abuse: cost in medical time and finance. Archives of disease in childhood 67.7
18. Fang, Xiangming, et al. (2015). The burden of child maltreatment in the East Asia and Pacific region. Child abuse \& neglect 42 (2015): 146162

19. Fang, Xiangming, et al. (2015). The burden of child maltreatment in China: a systematic review. Bulletin of the World Health Organization 93.3 (2015): 176-185C

20. Peterson, Cora, et al. (2014). The medical cost of abusive head trauma in the United States. Pediatrics 134.1 (2014): 91-99

21. Perryman Report (2014). An assessment of the Economic Cost of Child Maltreatment

22. Corso, Phaedra S., et al.(2007). Medical costs and productivity losses due to interpersonal and self-directed violence in the United States. American journal of preventive medicine 32.6 (2007): 474-48

23. Saied-Tessier, A. (2014). Estimating the costs of child sexual abuse in the UK. London: NSPCC.

24. Florence, Curtis, et al. (2013). Health care costs associated with child maltreatment: impact on Medicaid. Pediatrics 132.2 (2013): 312-318.

25. Kahui, Sherilee, and Suzanne Snively (2014). Measuring the Economic Costs of Child Abuse and Intimate Partner Violence to New Zealand. Glenn Inquiry.

26. Woodman J. (2011). Healthcare use by children fatally or seriously harmed by child maltreatment: analysis of a national case series 2005-2007. Arch Dis Child. 2011 Mar; 96(3):270-5. 
Table A1: Definition of Child Maltreatment Used in the UK Literature

\begin{tabular}{|c|c|c|c|}
\hline Study & Author & Data & Definition of maltreatment \\
\hline $\begin{array}{l}\text { Child Neglect and } \\
\text { Maltreatment and } \\
\text { Childhood-to-Adulthood } \\
\text { Cognition and Mental } \\
\text { Health in a Prospective Birth } \\
\text { Cohort. }\end{array}$ & Geoffroy MC & NCDS & $\begin{array}{l}\text { Neglect at } 7 \text { and } 11 \text { (parents and teacher): mother/father hardly ever } \\
\text { takes on outings, had little interest in the child's education, child looks } \\
\text { undernourished, scruffy/dirty. Neglect at age } 45 \text { (related to } 16 \text { years): I was } \\
\text { neglected, Mother/father not at all affectionate toward me. Abuse at age } \\
45 \text { (related to } 16 \text { years) covers psychological abuse, physical abuse, sexual } \\
\text { abuse and witnessing physical/sexual abuse. }\end{array}$ \\
\hline $\begin{array}{l}\text { Child maltreatment and } \\
\text { household dysfunction: } \\
\text { associations with pubertal } \\
\text { development in a British } \\
\text { birth cohort. }\end{array}$ & Li L & NCDS & $\begin{array}{l}\text { Neglect at } 7 \text { : mother/father hardly ever reads to child or takes on outings, } \\
\text { had little interest in the child's education, low parental aspiration. Abuse } \\
\text { at age } 45 \text { (physical, sexual, psychological), reported at } 45 \text { (related to 16). } \\
\text { Witnessing abuse, reported at } 45 \text { (related to 16). Neglect at age } 45 \text { (related } \\
\text { to 16): I was neglected, Mother/father not at all affectionate toward me. }\end{array}$ \\
\hline $\begin{array}{l}\text { The influence of childhood } \\
\text { adversity on social relations } \\
\text { and mental health at mid- } \\
\text { life. }\end{array}$ & Ford E & NCDS & $\begin{array}{l}\text { Neglected appearance at } 7 \text { and/or } 11 \text { : the medical examiner reported an } \\
\text { underfed/neglected. Parental physical abuse and sexual abuse reported } \\
\text { at } 45 \text { years. Cumulative adversity was measured by counting the reports of } \\
\text { neglected appearance, parental physical abuse, parental sexual abuse, but } \\
\text { also being in care, parental divorce, maternal and paternal absence. }\end{array}$ \\
\hline $\begin{array}{l}\text { Does the influence of } \\
\text { childhood adversity on } \\
\text { psychopathology persist } \\
\text { across the lifecourse? } \\
\text { A } 45 \text {-year prospective } \\
\text { epidemiologic study. }\end{array}$ & Clark C & NCDS & $\begin{array}{l}\text { Neglected appearance at } 7 \text { and/or } 11 \text { : the medical examiner reported an } \\
\text { underfed/neglected. Parental physical abuse and sexual abuse reported } \\
\text { at } 45 \text { years. Cumulative adversity was measured by counting the reports of } \\
\text { neglected appearance, parental physical abuse, parental sexual abuse, but } \\
\text { also being in care, parental divorce, maternal and paternal absence. }\end{array}$ \\
\hline
\end{tabular}

Obesity and type 2 diabetes Thomas C NCDS Neglect: little interest in child's education rated for each parent by risk in midadult life: the role of childhood adversity. teachers at 7, 11, 16; hardly ever has outings with mother or father at 7,11 ; mother or father hardly ever reads to the child at 7 ; low parental aspirations at 11 and 16 . Neglect at 7 and 11 (by teacher) "appearance of child" as scruffy or dirty. Emotional neglect at 45 (related to 16): how affectionate was mother/father. Physical neglect at 45 (related to 16): I was neglected. Abuse at 45 (related to 16): emotional, physical and sexual together. Witnessing abuse at 45 (related to 16). Received too much physical punishment defined as household dysfunction at 45 (related to 16)

\begin{tabular}{|c|c|c|c|}
\hline $\begin{array}{l}\text { Childhood maltreatment } \\
\text { and BMI trajectories to mid- } \\
\text { adult life: follow-up to age } \\
50 \text { y in a British birth cohort. }\end{array}$ & Power C & NCDS & $\begin{array}{l}\text { Neglect at } 7 \text { and } 11 \text { (parents and teacher): mother/father hardly ever } \\
\text { takes on outings, had little interest in the child's education, child looks } \\
\text { undernourished, scruffy/dirty. Abuse at age } 45 \text { (related to } 16 \text { years) covers } \\
\text { psychological abuse, physical abuse and sexual abuse. }\end{array}$ \\
\hline $\begin{array}{l}\text { Childhood adversity as a } \\
\text { risk for cancer: findings } \\
\text { from the } 1958 \text { British birth } \\
\text { cohort study. }\end{array}$ & $\begin{array}{l}\text { Kelly-Irving } \\
M\end{array}$ & & $\begin{array}{l}\text { Physical neglect at } 7,11,16 y \text { : child appears undernourished/dirty at } 7,11 \text {, } \\
16 y \text {. 'Adverse childhood experiences', however also include: being in care, } \\
\text { offenders, parental separation, mental illness, alcohol abuse. }\end{array}$ \\
\hline
\end{tabular}

\begin{tabular}{|c|c|c|c|}
\hline $\begin{array}{l}\text { Adverse childhood } \\
\text { experiences and } \\
\text { physiological wear-and-tear } \\
\text { in midlife: Findings from the } \\
1958 \text { BBC. }\end{array}$ & $\begin{array}{l}\text { Barboza } \\
\text { Solis C }\end{array}$ & NCDS & $\begin{array}{l}\text { Physical neglect ay } 7,11,16 y \text { : child appears undernourished/dirty at } 7,11 \text {, } \\
16 y \text {. 'Adverse childhood experiences', however also include: being in care, } \\
\text { offenders, parental separation, mental illness, alcohol abuse. }\end{array}$ \\
\hline $\begin{array}{l}\text { Adverse childhood } \\
\text { experiences and child-to- } \\
\text { adult height trajectories } \\
\text { in the } 1958 \text { British birth } \\
\text { cohort. }\end{array}$ & Denholm R & NCDS & $\begin{array}{l}\text { Neglect at } 7 \text { : mother/father hardly ever reads to child or takes on outings, } \\
\text { had little interest in the child's education, low parental aspiration. } \\
\text { Neglect at age } 45 \text { (related to } 16 \text { ): I was neglected, Mother/father not at } \\
\text { all affectionate toward me. Physical abuse, sexual abuse, psychological } \\
\text { abuse, reported at } 45 \text { (related to } 16 \text { ). Witnessing abuse, reported at } 45 \\
\text { (related to 16). }\end{array}$ \\
\hline
\end{tabular}




\begin{tabular}{|c|c|c|c|}
\hline Study & Author & Data & Definition of maltreatment \\
\hline $\begin{array}{l}\text { Child maltreatment and } \\
\text { household dysfunction in a } \\
\text { British birth cohort }\end{array}$ & Denholm R & NCDS & $\begin{array}{l}\text { Psychological (or emotional) abuse: Intentional behaviour that conveys to } \\
\text { a child that he/she is worthless, flawed, unloved, unwanted, endangered } \\
\text { or valued only in meeting another's needs. UK definition includes harmful } \\
\text { (unintentional) parent-child interactions: 'the persistent emotional } \\
\text { ill-treatment of a child such as to cause severe and persistent adverse } \\
\text { effects on the child's emotional development'. Verbally abused at age } 45 \\
\text { (related to 16): I was verbally abused by a parent ( } 45 \text { years); I suffered } \\
\text { humiliation, ridicule, bullying or mental cruelty from a parent. Physical } \\
\text { abuse: Intentional use of physical force or implements against a child } \\
\text { that results in, or has the potential to result in, physical injury. Physical } \\
\text { abuse at } 45 \text { (related to } 16 \text { ): I was physically abused by a parent - punched, } \\
\text { kicked or hit or beaten with an object, or needed medical treatment. Sexual } \\
\text { abuse: Any completed or attempted sexual act, sexual contact or non- } \\
\text { contact sexual interaction with a child by a caregiver. Sexually abused at } \\
45 \text { (related to } 16 \text { ): I was sexually abused by a parent. Witnessing intimate } \\
\text { partner violence: any incident of threatening behaviour, violence or abuse } \\
\text { (psychological, physical, sexual, financial or emotional) between intimate } \\
\text { partners or adult family members, irrespective of sex or sexuality. Witness } \\
\text { of violence at } 45 \text { (related to } 16 \text { ): I witnessed physical or sexual abuse } \\
\text { of others in my family. Neglect: Failure to meet a child's basic physical, } \\
\text { emotional, medical/dental or education need; failure to provide adequate } \\
\text { nutrition, hygiene or shelter; or failure to ensure a child's safety. Neglected } \\
\text { at age } 45 \text { (related to } 16 \text { ): I was neglected; Mother unaffectionate; Father } \\
\text { unaffectionate; Mother hardly ever reads to child ( } 7 \text { years); Father hardly } \\
\text { ever reads to child ( } 7 \text { years). }\end{array}$ \\
\hline $\begin{array}{l}\text { Childhood abuse is } \\
\text { associated with methylation } \\
\text { of multiple loci in adult DNA. }\end{array}$ & Suderman M & NCDS & $\begin{array}{l}\text { Abuse reported at } 45 \text { (related to } 16 \text { ). Emotional: I was verbally abused by } \\
\text { a parent; I suffered humiliation, ridicule, bullying or mental cruelty from a } \\
\text { parent. Physical: I was physically abused by a parent - punched, kicked or } \\
\text { hit or beaten with an object, or needed medical treatment. Sexual: I was } \\
\text { sexually abused by a parent. }\end{array}$ \\
\hline $\begin{array}{l}\text { Maternal childhood abuse } \\
\text { and offspring adjustment } \\
\text { over time. }\end{array}$ & Collishaw S & ALSPAC & $\begin{array}{l}\text { Maternal childhood experience of abuse (sexual, physical, and emotional), } \\
\text { retrospectively: "whether a parent had been physically or emotionally cruel } \\
\text { to them prior to age } 17 \text { years". }\end{array}$ \\
\hline $\begin{array}{l}\text { Child maltreatment in the } \\
\text { children of the nineties": a } \\
\text { cohort study of risk factors." }\end{array}$ & $\begin{array}{l}\text { Sidebotham } \\
P\end{array}$ & ALSPAC & $\begin{array}{l}\text { Social Services child protection registers were screened for any children } \\
\text { with birth dates who have been investigated for possible child abuse or } \\
\text { neglect. Physical injury: actual or likely physical injury to a child, or failure } \\
\text { to prevent physical injury (or suffering) to a child including deliberate } \\
\text { poisoning, suffocation and Munchausen syndrome by proxy. Neglect: } \\
\text { persistent/severe neglect of a child, or failure to protect a child from } \\
\text { exposure to any kind of danger, including cold or starvation, or extreme } \\
\text { failure to carry out important aspects of care, resulting in significant } \\
\text { impairment of the child's health or development, including non-organic } \\
\text { failure to thrive. Sexual abuse: actual or likely sexual exploitation of a } \\
\text { child or adolescent. The child may be dependent and/or developmentally } \\
\text { immature. Emotional abuse: actual or likely severe adverse effect on the } \\
\text { emotional and behavioural development of a child caused by persistent } \\
\text { or severe emotional ill-treatment or rejection. All abuse involves some } \\
\text { emotional ill-treatment. }\end{array}$ \\
\hline
\end{tabular}




\begin{tabular}{|c|c|c|c|}
\hline Study & Author & Data & Definition of maltreatment \\
\hline $\begin{array}{l}\text { Child maltreatment in the } \\
\text { children of the 90s: the } \\
\text { child. }\end{array}$ & $\begin{array}{l}\text { Sidebotham } \\
\text { P. }\end{array}$ & ALSPAC & $\begin{array}{l}\text { Social Services child protection registers were screened for any children } \\
\text { who have been investigated for possible child abuse or neglect. }\end{array}$ \\
\hline $\begin{array}{l}\text { Child maltreatment in } \\
\text { the Children of the } 90 \mathrm{~s} \text { : } \\
\text { deprivation. }\end{array}$ & $\begin{array}{l}\text { Sidebotham } \\
\text { P. }\end{array}$ & ALSPAC & $\begin{array}{l}\text { Social Services child protection registers were screened for any children } \\
\text { who have been investigated for possible child abuse or neglect. }\end{array}$ \\
\hline $\begin{array}{l}\text { Child maltreatment in } \\
\text { the children of the } 90 \text { s: } \\
\text { a longitudinal study of } \\
\text { parental risk factors. }\end{array}$ & $\begin{array}{l}\text { Sidebotham } \\
\text { P. }\end{array}$ & ALSPAC & $\begin{array}{l}\text { Social Services child protection registers were screened for any children } \\
\text { who have been investigated for possible child abuse or neglect. }\end{array}$ \\
\hline $\begin{array}{l}\text { Measuring adolescents' } \\
\text { exposure to victimization: } \\
\text { the Environmental Risk } \\
\text { (E-Risk) Longitudinal Twin } \\
\text { Study }\end{array}$ & $\begin{array}{l}\text { Fisher, Helen } \\
\text { L. }\end{array}$ & E-Risk & Sexual victimization, family violence, maltreatment, neglect. \\
\hline $\begin{array}{l}\text { Childhood Trauma and } \\
\text { Children's Emerging } \\
\text { Psychotic Symptoms: } \\
\text { A Genetically Sensitive } \\
\text { Longitudinal Cohort Study }\end{array}$ & Arseneault L & E-Risk & $\begin{array}{l}\text { Maltreatment by an adult by interviewing mothers. Do you remember when } \\
\text { the child was disciplined severely enough that } \mathrm{s} / \text { he may have been hurt? } \\
\text { Did you worry that you or someone else may have harmed or hurt the child } \\
\text { during those years? }\end{array}$ \\
\hline $\begin{array}{l}\text { The limits of child effects: } \\
\text { evidence for genetically } \\
\text { mediated child effects } \\
\text { on corporal punishment } \\
\text { but not on physical } \\
\text { maltreatment. }\end{array}$ & Jaffee SR & E-Risk & $\begin{array}{l}\text { Corporal punishment and child physical maltreatment. Whether the } \\
\text { child had experienced a variety of disciplinary practices in the past } \\
\text { year, some of which assessed nonphysical discipline (e.g., "isolation," } \\
\text { "withdrew privileges") and three of which assessed corporal punishment } \\
\text { ("grabbing or shaking," "smacking or hitting," or engaging in "other physical } \\
\text { discipline"). Probe questions about potential physical maltreatment, } \\
\text { including "When [name] was a toddler, do you remember any time when } \\
\text { she/he was disciplined severely enough that she/he may have been } \\
\text { hurt?" and "Did you worry that you or someone else [such as a babysitter, } \\
\text { a relative, or a neighbour] may have harmed or hurt [name] during those } \\
\text { years?" }\end{array}$ \\
\hline $\begin{array}{l}\text { Variation in recorded child } \\
\text { maltreatment concerns in } \\
\text { UK primary care records: } \\
\text { a cohort study using The } \\
\text { Health Improvement } \\
\text { Network (THIN) database. }\end{array}$ & Woodman J & THIN & $\begin{array}{l}\text { "Maltreatment-related" codes. 1. Child protection procedures. Codes } \\
\text { indicating child protection plan, case conference, or child protection } \\
\text { investigation. 2. Direct references to maltreatment or out-of-home- } \\
\text { care. Codes making explicit reference to abuse or neglect (including } \\
\text { domestic violence) or to formal out-of-home care. 3. High risk child. Codes } \\
\text { indicating high levels of social welfare need or concern in the child or } \\
\text { family, including a history of abuse of neglect. } 4 \text {. Contact with children's } \\
\text { social care. Codes indicating that the child is involved with or has been } \\
\text { referred to children's social care (not including codes specifically referring } \\
\text { to 'child protection procedure' codes. }\end{array}$ \\
\hline
\end{tabular}


Table A2: Supplementary NCDS Results of the Effects of the Global Measure of CM at Sweep 4 (Age 23)

\begin{tabular}{llllll}
\hline & $\begin{array}{l}\text { Labour Market } \\
\text { Outcome: } \\
\text { Employed }\end{array}$ & $\begin{array}{l}\text { Healthy } \\
\text { Behaviours: } \\
\text { Current Smoker }\end{array}$ & $\begin{array}{l}\text { Mental Health } \\
\text { Problem: Any }\end{array}$ & $\begin{array}{l}\text { Mental Health } \\
\text { Problem: Neurotic } \\
\text { disorder }\end{array}$ & $\begin{array}{l}\text { Mental Health Problem: } \\
\text { Depression }\end{array}$ \\
\hline Any abuse & -0.0202 & 0.0229 & 0.00592 & $0.00851^{*}$ & -0.00103 \\
\hline & $(0.0147)$ & $(0.0201)$ & $(0.00512)$ & $(0.00478)$ & $(0.00526)$ \\
\hline
\end{tabular}

${ }^{* \star *} p<0.01,{ }^{* \star} p<0.05,{ }^{*} p<0.1$

Note: The table contains estimates from linear regression models with robust standard errors. Each cell contains results from a different model. Within each cell, we first report the estimated coefficients; we then report in parentheses robust standard errors; we last report in italics the sample size. Controls are included for: (1) a basic set of demographics (gender, ethnicity and interview date); (2) an extended set of demographics recorded at birth (social class of the husband and of the father of the mother, parity, birthweight, whether the mother stayed in school beyond the minimum school leaving age, marital status, smoking in pregnancy, mother's and father's age at the child's birth, working during pregnancy, weight before pregnancy, mother's height, whether the mother had any antenatal visit in the first trimester of pregnancy, and the number of people per room); (3) other early adverse childhood experiences, as collected in the age 7 sweep (whether the child lived with both natural parents, whether there was any family contacts with the probation officer, whether the parents divorced, whether a parent was alcoholic, whether the death of the father or of the mother occurred, whether there was any domestic tension, whether there were financial or housing difficulties, and the number of times the family moved since the birth of the child); and, (4) an extended set of early adverse childhood experiences, reported retrospectively in the biomedical sweep (whether the mother suffered from nervous or emotional trouble, whether the mother had trouble drinking, whether there was some or a lot of conflict and tension there was in the house, whether the child grew up in poverty or in financial hardship).

a Neurotic disorder: anxiety states, panic attacks, nerves, nervous breakdown, phobic state, obsessive-compulsive disorders, hypochondria, agoraphobia, unspecified neuroses). 
Table A3: Measures included in the datasets considered in the quantitative analysis of the effect of child maltreatment

\begin{tabular}{|c|c|c|c|c|c|c|}
\hline & & BHPS \& UKHLS & & ELSA & ESYTC & NCDS \\
\hline \multicolumn{7}{|l|}{ Exposures } \\
\hline Abuse - Sexual & & & & & $\begin{array}{l}\text { W3 }(C+S W+P) \\
W 4(C+P)\end{array}$ & $R(45 Y)$ \\
\hline Abuse - Physical & & $\mathrm{P}(\mathrm{W} 12, \mathrm{~W} 13, \mathrm{~W} 17)$ & $P(W 3, W 4, W 5)$ & W3 (R) & $\begin{array}{l}\text { W3 }(C+S W+P) \\
W 4(C+P)\end{array}$ & $R(45 Y)$ \\
\hline Abuse-Emotional & & $P(W 12, W 13, W 17)$ & P (W3,W4,W5) & & & $R(45 Y)$ \\
\hline Neglect & & $P(W 12, W 13, W 17)$ & $P(W 3, W 4, W 5)$ & W3 (R) & $\begin{array}{l}\text { W3 }(C+S W+P), \\
W 4(C+P)\end{array}$ & $R(45 Y)$ \\
\hline \multicolumn{7}{|l|}{ Outcomes } \\
\hline \multirow[t]{3}{*}{ Health - Mental } & Any type & & & W1-W4 & W1,W2,W3,W4 & 33Y,42Y,50Y,55Y \\
\hline & Anxiety & & & W1-W4 & & $33 Y, 42 Y, 50 Y, 55 Y$ \\
\hline & Depression & W12-W18 & W2-W5 & W1-W4 & W1,W2,W3,W4 & $33 Y, 42 Y, 50 Y, 55 Y$ \\
\hline \multirow[t]{4}{*}{ Health - Physical } & Obesity & W14,W16,W18 & W2-W5 & W1-W4 & & $33 Y, 42 Y, 50 Y, 55 Y$ \\
\hline & Hypertension & & & W1-W4 & & $33 Y, 42 Y, 50 Y, 55 Y$ \\
\hline & Diabetes & & & W1-W4 & & $33 Y, 42 Y, 50 Y, 55 Y$ \\
\hline & Cancer & & & W1-W4 & & $33 Y, 42 Y, 50 Y, 55 Y$ \\
\hline \multirow[t]{2}{*}{$\begin{array}{l}\text { Unhealthy } \\
\text { behaviours }\end{array}$} & Alcohol Use & W15,W17,W18 & W5 & W1-W4 & W1,W2,W3,W4 & $33 Y, 42 Y, 50 Y, 55 Y$ \\
\hline & Smoking & & W5 & W1-W4 & W1,W2,W3,W4 & $33 Y, 42 Y, 50 Y, 55 Y$ \\
\hline Disability & Disability benefits & & & W1-W4 & & \\
\hline \multirow[t]{2}{*}{ Productivity } & Employment & & W2-W5 & W1-W4 & W4 & 33Y,42Y,50Y,55Y \\
\hline & Earnings & & W2-W5 & W1-W4 & & $33 Y, 42 Y, 50 Y, 55 Y$ \\
\hline
\end{tabular}

Note: We report the age in the case of the NCDS since it is a cohort, and the wave for the other datasets since at each wave there are respondents of different ages. Abbreviations: $W=$ wave; $Y=y e a r s ; C=c h i l d ; S W=$ social worker; $R=$ retrospective; $P=$ proxy. $B H P S=B r i t i s h$ Household Panel Survey; UKHLS=Understanding Society; ELSA=English Longitudinal Study of Aging; ESYTC=Edinburgh Study of Youth Transitions and Crime; NCDS=National Child Development Study. 
Find out more about our work at nspcc.org.uk 